\title{
Analytical and numerical investigation of nonlinear internal gravity waves
}

\author{
S. P. Kshevetskii \\ Kaliningrad State University, Kaliningrad, Russia
}

Received: 22 June 1999 - Accepted: 25 April 2000

\begin{abstract}
The propagation of long, weakly nonlinear internal waves in a stratified gas is studied. Hydrodynamic equations for an ideal fluid with the perfect gas law describe the atmospheric gas behaviour. If we neglect the term $\rho d w / d t$ (product of the density and vertical acceleration), we come to a so-called quasistatic model, while we name the full hydro-

ence of difference interval finiteness on a numerical solution is investigated. It is revealed that a numerical viscosity and numerical dispersion can play the role of regularizators to a nonlinear quasistatic problem. To avoid this effect, the grid steps should be taken less than some threshold values found theoretically.
\end{abstract} dynamic model as a nonquasistatic one. Both quasistatic and nonquasistatic models are used for wave simulation and the models are compared among themselves. It is shown that a smooth classical solution of a nonlinear quasistatic problem does not exist for all $t$ because a gradient catastrophe of nonlinear internal waves occurs. To overcome this difficulty, we search for the solution of the quasistatic problem in terms of a generalised function theory as a limit of special regularised equations containing some additional dissipation term when the dissipation factor vanishes. It is shown that such solutions of the quasistatic problem qualitatively differ from solutions of a nonquasistatic nature. It is explained by the fact that in a nonquasistatic model the vertical acceleration term plays the role of a regularizator with respect to a quasistatic model, while the solution qualitatively depends on the regularizator used. The numerical models are compared with some analytical results. Within the framework of the analytical model, any internal wave is described as a system of wave modes; each wave mode interacts with others due to equation nonlinearity. In the principal order of a perturbation theory, each wave mode is described by some equation of a KdV type. The analytical model reveals that, in a nonquasistatic model, an internal wave should disintegrate into solitons. The time of wave disintegration into solitons, the scales and amount of solitons generated are important characteristics of the nonlinear process; they are found with the help of analytical and numerical investigations. Satisfactory coincidence of simulation outcomes with analytical ones is revealed and some examples of numerical simulations illustrating wave disintegration into solitons are given. The phenomenon of internal wave mixing is considered and is explained from the point of view of the results obtained. The numerical methods for internal wave simulation are examined. In particular, the influ-

\section{Introduction}

The majority of large-scale atmospheric and ocean models, a priori, assume a local hydrostatic equilibrium. The approximation corresponding to this supposition consisting of the term of a vertical acceleration is omitted is called a hydrostatic or quasistatic approximation. Richardson has suggested this simplification in 1922. One usually justifies the quasistatic approach by a small ratio of vertical and horizontal scales of large-scale fields; $\beta$ will denote the ratio of vertical and horizontal scales.

This justification seems quite convincing and is conventional now. Nevertheless, some mathematical research reveals that the passage from a full hydrodynamic model to the limit of a quasistatic model is absent (Long, 1965; Kshevetskii and Leble, 1985, 1988). The reason for this strange phenomenon is concealed in equation nonlinearity. Within the framework of a nonlinear hydrostatic model, at the wave front, horizontal gradients ordinarily increase with time, together with vertical accelerations, which can reach infinite values over time. That is, over time, depending on initial conditions, the solution of a hydrostatic problem becomes unexistant.

The unpleasant fact would be absent if the quasistatic approach was not used. It is necessary to keep only the vertical acceleration term in the equations. Actually, it is an unjustified optimism. When developing a nonquasistatic model, we encounter a number of specific mathematical difficulties.

Let us describe briefly some decisions, which should be made when developing a nonquasistatic model. In such a model exists a sound wave mode. Sound wave frequencies 
are huge in comparison with internal wave frequencies. The sound waves represent a fast mode, while the internal waves represent a slow mode. The error of any numerical method is expressed through higher derivatives of the solution. The greater these higher derivatives are, the greater the simulation error. All fast-oscillating functions have large derivatives. Therefore, high-frequency waves significantly contribute to the error and prevent numerical simulating of a slow mode.

The difficulty described cannot be overcome at the expense of the diminution of difference of grid steps. On the contrary, the diminution of grid steps can only contribute to the aggravation of the outcome. Fortunately, mathematical tools are able to overcome the difficulty described. Some special numerical methods are indifferent in relation to a fast mode (Kshevetskii, 1990, 1998). These methods may be called uniformly converging methods because they meet the requirement that the convergence is uniform in $\beta$. The uniformly converging numerical methods are difficult ones, but they have an advantage. The errors originated from a fast mode are not accumulated over time. The methods allow one to work with the equations as if the model is a hydrostatic one.

The obstacles described above do not exhaust all difficulties. To describe the new difficulties easier, let us return to the consideration of a quasistatic problem. As it has been mentioned, a classical smooth solution of the nonlinear quasistatic problem may be unexisting for large $t$. Such cases are not rare, but it depends on initial conditions. We take an interest in the fluid behaviour for large $t$. Therefore, it is necessary somehow to restore correctness of the problem for all $t$.

It is natural to try to restore the problem correctness via a conventional reception, searching for a solution in terms of generalised functions (Richtmyer, 1978). It is usual practice in a nonlinear theory. Often the generalised solution is obtained as follows. At first, one adds into the equations a special term with a preceding small parameter. The newly modified equations are called regularised equations. Then one solves the regularised equations and takes the limit when the small parameter tends to zero; thus the term containing the small parameter vanishes. If, in doing so, the limit solution exists, it is called a generalised solution of the problem (Lions, 1969).

A shortcoming of the regularisation technique is that the generalised solution obtained depends on the regularisation used. In this sense, the solution is not unique. Therefore, it is very important to take the regularisation correctly. The small term $\rho \partial w / \partial t$ in the nonhydrostatic model may be considered as a natural regularizator. This term, in fact, fulfills the role of a regularizator because it ensures the existence of a smooth solution. However, we immediately should notice that in such conditions the effects of the numerical dissipation and numerical dispersion are able to play the role of a regularizator, instead of the term $\rho \partial w / \partial t$. These numerical effects always take place in all numerical models due to the finiteness of difference intervals. The competition between different regularizators is rather probable in this case with the effects of the numerical dissipation and numerical dispersion against the term $\rho \partial w / \partial t$. Various regularizators result in various generalised solutions. Therefore, the situation requires detail study.

One can say that a nonquasistatic model is studied in the paper. However, it is more correct to consider this investigation differently. In this work, a usual quasistatic model is considered and various regularisation techniques are investigated. The nonquasistatic model is considered, but only as one of the mathematically possible regularisations. This regularisation is more justified physically, and, consequently, is examined more carefully.

It is necessary to notice that the issue of equation regularisation is insufficiently developed for the present. As far as the author can view the question, originally it was presumed that parabolic regularisations are only physically admissible, and that the solution weakly depends on the regularisation used. Von Neumann and Richtmyer have suggested the parabolic regularisation in 1950. Later, it was discovered that the solution can depend on the regularisation used, but only dissipation terms were allowed as regularizators (Samarskiy and Popov, 1980). In the problem under consideration, a dispersion term plays the role of a regularizator. The situation is unusual and the author could not carry out a mathematically finished investigation adequate for all practical needs of geophysics. Nevertheless, in the paper some outcomes of several test simulations illustrating efficiency of the constructed mathematical model are shown. An effect of internal wave mixing is considered as well, as it is mathematically associated with considered matters.

Dissipation effects are not taken into account. The problem under consideration is two-dimensional. The simplifications are not adopted because of essential difficulties. A more complete model could take dissipation and three-dimensionality into consideration. However, within the framework of the present research, the "superfluous" terms are cut off in order to concentrate on the investigation of critical matters.

\section{Basic equations and suppositions being used}

The scale height $H$ of the atmosphere depends on height $z$ above the Earth's surface. At the height of $80-90 \mathrm{~km}$ the ultimate velocity of internal wave propagation in the atmosphere reaches a minimum. Therefore, the internal waves with propagation velocities greater than $\sqrt{4(\gamma-1) g H_{\min } / \gamma}$ can not intercross the turbopause region and therefore they propagate along the Earth's surface horizontally. In relation to these waves, one can consider the atmosphere as a waveguide.

To simplify the model, we consider a gas exponentially stratified in density. We shall ensure wave-guide propagation of internal waves at the expense of the rigid cover conditions: $w(x, z=0, t)=w(x, z=h, t)=0$. Here $h$ is an effective thickness of the atmospheric wave-guide, $w$ is the vertical velocity, $x$ and $z$ are the horizontal and vertical coordinates. The upper boundary condition qualitatively takes 
into account a wave reflection effect by the turbopause region. The lower boundary condition is natural.

We suppose that the atmospheric gas behaviour is governed by $2 \mathrm{D}$ hydrodynamic equations for an ideal fluid with the perfect gas law:

$$
\begin{aligned}
& \frac{d \rho}{d t}+\rho \nabla \cdot \boldsymbol{V}=0, \\
& \rho \frac{d u}{d t}=-\frac{\partial P}{\partial x}, \\
& \rho \frac{d w}{d t}=-\frac{\partial P}{\partial z}-\rho g, \\
& \frac{c_{v}}{\mu} \rho \frac{d T}{d t}=-P \nabla \cdot \boldsymbol{V}, \quad P=\frac{\rho R T}{\mu} .
\end{aligned}
$$

The labels are conventional; no special explanations are required. The boundary conditions have been noted above.

We shall study, for the most part, long internal gravity waves; $\beta=k_{x} l_{z} \ll 1$ for long waves. Here $k_{x}^{-1}$ denotes a characteristic horizontal scale of the wave. The vertical scale is $l_{z}=\min \left(k_{z}^{-1}, H\right)$, where $k_{z}$ is a typical value of a vertical component of the wave vector. Using the dispersion relation for internal gravity waves, one can easily deduce the estimate $\omega \sqrt{H / g} \approx k_{x} l_{z}=\beta \ll 1$. The symbols $\omega$ and $g$ denote the wave frequency and gravity acceleration. With the help of polarisation relations for internal waves, one can easily obtain the following estimates:

$\frac{u}{\sqrt{G H}} \sim \frac{w}{\beta \sqrt{g H}} \sim \frac{\Delta P}{P_{0}} \sim \frac{\Delta \rho}{\rho_{0}} \sim \sigma$

Here the parameter of nonlinearity $\sigma=\Delta P / P_{0} \ll 1 ; \Delta P$, $\Delta \rho$ are the amplitudes of the pressure and density variations on account of wave propagation, $P_{0}, \rho_{0}$ are the background pressure and density of the unperturbed atmosphere.

Some elementary estimates reveal that the vertical accelerations of fluid particles are small for internal waves. It means that $\rho d w / d t \sim \rho_{0} \beta^{2} \sigma g$ is much less than $\left(\rho-\rho_{0}\right) g \sim \sigma \rho_{0} g$ and we can consider $\rho d w / d t$ in the third equation of system (1) as small. In dimensionless variables, this term is of the order $O\left(\beta^{2}\right)$. All other linear terms are of the identical order $O(1)$. The nonlinear terms are of the orders $\sigma, \sigma^{2}, \sigma \beta^{2}$. If we neglect $\rho d w / d t$, we use a so-called quasistatic approximation. This approximation is very popular in geophysics. At present, it is a fundamental equation of the dynamic meteorology.

The curvature of the Earth's surface, the Earth's rotation, and dissipation is not taken into account. Some estimates reveal that these effects are not of value to resolve for nonlinear processes under consideration. Some notes concerning these effects will be made below.

\section{Analytical model of nonlinear internal gravity waves}

Equations (1) are very complex ones. At present, such challenges may only be solved numerically. It is possible to solve (1) by a Galerkin method (Fletcher, 1984). In the framework of a Galerkin method, a solution is sought after as a generalised Fourier series with solution expansion on any complete set of basis functions. For example, a solution of equations (1) for the horizontal velocity can be presented as the generalised Fourier series $u(x, x, t)=\sum_{n} \Theta^{n}(x, t) S^{n}(z)$ on some complete set of functions $S^{n}(z)$. Here $\Theta^{n}(x, t)$ are factors of the Fourier series. One can take the functions $S^{n}(z)$ arbitrarily, if they only form a complete set. It is very convenient in the problem under consideration to take an $S^{n}(z)$ those functions arise at solving the linearized equations (1) by a method of separation of variables.

To be sure, the solution of system (1) can be written in a linear approximation as $\sum_{n} \Theta^{n}(x, t) S^{n}(z)$ as well. In a linear case, the solution can be constructed by a Fourier method of separation of variables. In doing so, the product $\Theta^{n}(x, t) S^{n}(z)$ is often named an eigen-mode of the problem, where $n$ denotes the mode number. The functions $S^{n}(z)$ describe a vertical shape of the mode. The functions $S^{n}(z)$ satisfy some Sturm-Liouville boundary value problem on eigenvalues. This Sturm-Liouville problem automatically arises when solving the linearized equations (1) by a method of separation of variables.

In a nonlinear case, we shall call the product $\Theta^{n}(x, t) S^{n}(z)$ a mode, by analogy to a linear theory. To supplement the Galerkin method with some "valid" simplifications based upon the smallness of $\sigma, \beta$, then the set of equations (1) can be rewritten with the error $O\left(\sigma^{2}+\beta^{4}\right)$ as follows (Kshevetskii and Leble, 1985, 1988):

$$
\begin{aligned}
\Theta_{t}^{n}+ & c_{n} \Theta_{x}^{n}+\frac{\sigma}{2} \sum_{m, l} F_{l m}^{n} \Theta^{l} \Theta_{x}^{m}+\frac{\beta^{2}}{2}\left(\frac{\gamma-c_{n}^{2}}{\gamma-1} c_{n}^{3} \Theta_{x x x}^{n}\right. \\
& \left.+\frac{\gamma+c_{n}^{2}}{\gamma-1} c_{n}^{3} \Theta_{x x x}^{-n}\right)=0 .
\end{aligned}
$$

Some modern derivation of equations (2) is given in Appendix A.

The factor $c_{n}$ in (2) is the propagation velocity of mode $n$ in a linear approximation. The waves with $n>0$ propagate to the right and the waves with $n<0$ propagate to the left, $c_{-n}=-c_{n} . F_{l m}^{n}$ are the constants defining the effectiveness of nonlinear interaction of modes. They are expressed by rather bulky integrals, whose integrands contain functions $S^{n}(z), S^{l}(z), S^{m}(z)$ and their derivatives. Equations (2) are written down in dimensionless variables. That is, $x$ is the dimensionless horizontal coordinate and $t$ is time: $x_{\text {dimens. }}=(H / \beta) x_{\text {nondimens. }}, t_{\text {dimens. }}=\beta^{-1} \sqrt{H / g} t_{\text {nondimens. }}$.

The nonlinear terms in (2) are due to nonlinear terms in (1). The dispersion terms $\left(\beta^{2} / 2\right)\left(\gamma-c_{n}^{2}\right) /(\gamma-1) c_{n}^{3} \Theta_{x x x}^{n}$ and $\left(\beta^{2} / 2\right)\left(\gamma+c_{n}^{2}\right) /(\gamma-1) c_{n}^{3} \Theta_{x x x}^{-n}$ originate from the term $\rho_{0} \partial w / \partial t$ contained in the third equation of system (1). They take into account small deviations from the local hydrostatic equilibrium. Let us consider explicitly why the third derivatives have arisen there. To begin with, we make note that there is no necessity to calculate the small terms precisely. It is enough to use the principal order of a perturbation theory. We can neglect nonlinear terms and the term $\rho \partial w / \partial t$ in (1) in the dominant order. At that time, we see that $w$ 
is written via $\Theta^{n}(x, t)$ by means of a differentiation operation: $w(x, z, t)=\sum_{n}\left(\partial \Theta^{n}(x, t) \partial x\right) S^{n}(z)$ and $\Theta^{n}(x, t)=$ $f^{n}\left(x-c_{n} t\right)$. The functions $f^{n}$ are arbitrary at this approximation. Particular shapes of $S^{n}(z)$ are not important for common reasoning and, consequently, the functions $S^{n}(z)$ are not written down here. One differentiation operation is contained in $\rho \partial w / \partial t$. In deriving (2), the third equation of system (1) has to be differentiated one time with respect to $x$. So, the third derivatives of $\Theta^{n}$ have arisen in (2).

Applying $\sigma=0$ and $\beta=0$, we shall come to a known outcome of a linear theory of long internal waves: each wave mode $n$ propagates with the eigen velocity $c_{n}$ without changing the form. Therefore, it is possible to say that equations (2) describe the nonlinear wave as a system of modes; each mode interacts with others.

Boundary conditions influence the functions $S^{n}(z)$ and, subsequently, nonlinear constants $F_{l m}^{n}$. For any homogeneous boundary conditions, internal wave propagation is described by equations such as (2). In this sense, the particular aspect of boundary conditions is not important when we are interested in qualitative nonlinear effects. It is known that long internal gravity waves propagate almost horizontally. So, if a long internal gravity wave has been excited not far from the Earth's surface, this wave reaches the wave guide upper boundary only at large times: $t_{\text {dimens. }} \approx h /(\beta \sqrt{g H})$. Therefore, system (2) is suitable also to model non-waveguide propagation of internal waves, but only until waves have reached the height $h$. We see that the boundary conditions do not influence the outcomes critically. For this reason, we have made only a slight consideration of the choice of boundary conditions.

If we neglect the interaction of various wave modes and if we take only one of them presuming that only one wave mode was originally excited, we shall obtain a KdV model of nonlinear internal waves. The KdV model has been suggested for the studying of nonlinear internal waves in the mid 1960 's, in a stationary variant of the KdV equation (Long, 1965; Benjamin, 1966). Between 1970 and 1980, this model was developed and was adjusted to explain various atmospheric and ocean processes. In contrast to the classical $\mathrm{KdV}$ model, equations (2) allows one to consider immediately a number of wave modes. Each of them interacts with the others. Although equations (2) do not contain $z$, the vertical wave propagation is taken into account in the model just as it takes place in Fourier or Calerkin methods. The history of development of a $\mathrm{KdV}$ model of internal waves is given briefly in Appendix B. Some references to primary sources are given there as well.

As system (2) is of extreme interest from a physical point of view, attempts were undertaken repeatedly to integrate (2) precisely. At present, some precise integrable cases are found for two- and three-wave systems. One can be found in (Gürses and Karasu, 1996, 1998), with exhaustive information on exact integrable $\mathrm{KdV}$ systems and a number of references.

In the papers by the author and Leble $(1985,1988)$, some nonsingular perturbation theory was developed to solve (2), and an approximate solution to this system was obtained. The approximate solution is:

$$
\begin{aligned}
& \Theta^{n}(x, t) \approx \Theta_{0}^{n}(x, t)-\frac{\sigma}{2} \int_{0}^{t} \sum_{\substack{m, l \\
m \neq n \neq l}} \\
& F_{l m}^{n} \Theta_{0}^{l}\left(x-c_{n}\left(t-t^{\prime}\right), t^{\prime}\right) \Theta_{0 x}^{m}\left(x-c_{n}\left(t-t^{\prime}\right), t^{\prime}\right) d t^{\prime} \\
& \quad-\frac{\beta^{2}}{2} \int_{0}^{t} \frac{\gamma+c_{n}^{2}}{\gamma-1} c_{n}^{3} \Theta_{0 x x x}^{-n}\left(x-c_{n}\left(t-t^{\prime}\right), t^{\prime}\right) d t^{\prime} .
\end{aligned}
$$

The functions $\Theta_{0}^{l}(x, t)$ are solutions of Korteweg-de Vries type equations (Lamb, 1980), such as

$$
\begin{aligned}
\Theta_{0 t}^{n} & +c_{l} \Theta_{0 x}^{n}+\frac{\sigma}{2} F_{n n}^{n} \Theta_{0}^{n} \Theta_{0 x}^{n} \\
& +\frac{\beta^{2}}{2}\left(\frac{\gamma-c_{n}^{2}}{\gamma-1} c_{n}^{3}\right) \Theta_{0 x x x}^{n}=0 .
\end{aligned}
$$

The initial conditions are posed so: $\Theta_{0}^{n}(x, 0)=\Theta^{n}(x, 0)$.

On the right of (3), the addend takes into account nonlinear interaction of various modes. The nonlinear interaction of each wave mode with itself is taken into account immediately by $\Theta_{0}^{n}(x, t)$. The approximate solution takes into account various mode interactions and mode self-actions with inequality in rights. It takes place because each mode is in charge with respect to others; it loosens the interactions of the different modes. The interactions of various modes become apparent only at impacts of modes when wave carriers are intersected. At the same time, the nonlinear self-actions of modes continuously take place and are loosened by nothing. Therefore, they give the effects that are more essential. In Kshevetskii and Leble $(1985,1988)$, it was shown that the contributions of interaction of modes $n, m$ are proportionate to $\sigma /\left(c_{n}-c_{m}\right)$. For higher modes, the residuals $\left|\left(c_{n}-c_{m}\right)\right|$ are small and the mode interactions are already necessary for taking into account in the principal order of the perturbation theory. The last term in the right-hand side of (3) takes into account some small dispersion effects. In principle, this term can be excluded, having made some appropriate compensatory amendments in $\Theta_{0}^{n}(x, 0)$.

\section{Analysis of the analytical solution}

It is well known that at $t \rightarrow \infty$, the wave described by a $\mathrm{KdV}$ equation disintegrates into solitons and a wave tail of a comparatively small amplitude propagates behind the basic soliton wave (Lamb, 1980). The internal waves are approximately described by equations of a KdV type. Hence, internal gravity waves should behave like waves governed by a $\mathrm{KdV}$ equation: at $t \rightarrow \infty$, they should disintegrate into several soliton waves with a small-amplitude wave tail propagating behind. Main wave energy accumulates in the head part consisting of solitons. By understanding the internal wave behaviour, we can now formulate some questions of physical interest. The time of wave disintegration into solitons, the scales and amount of solitons generated are important characteristics of the nonlinear process. The physical sense 
of these soliton waves is of interest as well. What do these solitons represent? Are they vortices, domains of increased or decreased pressure, something similar to a high-frequency sound wave, or something other? Now we put aside these questions, and we undertake the investigation of some extreme cases of interest.

The case $\beta=0$ is analytically analysable as well. Moreover, this case is of special interest because it answers a socalled quasistatic approach; the quasistatic equation is used now as a fundamental equation of the atmosphere. Richardson has offered the quasistatic approximation in 1922. In terms of primitive equations (1), this approximation means that the term $\rho d w / d t$ in the equation for a vertical momentum is omitted, and the third equation of system (1) turns into a quasistatic one

$$
\frac{\partial P}{\partial z}+\rho g=0 .
$$

In dimensionless variables, the small discarded term $\rho d w / d t$ is of the order $O\left(\beta^{2}\right)$. The limit $\beta \rightarrow 0$ is also interesting because it corresponds to

$t_{\text {dimens. }}=\frac{1}{\beta} \sqrt{\frac{H}{g}} t_{\text {nondimens. }} \rightarrow \infty$.

That is, studying the limit, we can see also what happens for large $t_{\text {dimens. }}$.

In terms of equations (2), at $\beta=0$ we have a system of quasilinear equations:

$\Theta_{t}^{n}+c_{n} \Theta_{x}^{n}+\frac{\sigma}{2} \sum_{m, l} F_{l, m}^{n} \Theta^{l} \Theta_{x}^{m}=0$

With the error $O\left(\sigma^{2}\right)$, this system is equivalent to the hydrodynamic equations in the quasistatic approximation. It is well known that some waves governed by quasilinear equations are able to break. More precisely, the solution may become ambiguous over time. Taking this note into account, the equations (5) were carefully investigated in Kshevetskii and Leble $(1985,1988)$ and Kshevetskii (1998). The condition of wave breaking is $F_{n n}^{n} \Theta^{n}(x, t=0) \geq 0$. (This condition can be obtained directly from (3), (4 ).) The primitiveness of this condition reveals that the wave breaking cannot be an unusual event. If we assume that positive and negative values of $\Theta^{n}(x, t=0)$ are equiprobable, then, roughly speaking, the wave breaking must occur in one half of the events, at a large enough $t$. The wave breaking demonstrates the fact that an unambiguous smooth solution (classical solution) of the nonlinear quasistatic problem may be nonexistant

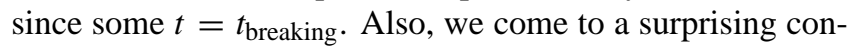
clusion: at $t_{\text {dimens. }} \rightarrow \infty$, the solution of the hydrodynamic equations (1) does not tend in general to reach the state of a mechanical equilibrium.

The smooth, classical solution of the nonlinear quasistatic problem does not exist for all $t$. To overcome this difficulty, it is natural to try to consider the solution in terms of the theory of generalised functions (Richtmyer, 1978). For nonlinear equations, one ordinarily calculates it in the following way
(Lions, 1969). At first, one adds into the equations any small term ensuring the existence of a smooth solution for all $t$ (5). This additional term is especially selected. The special term is called a regularizator and the revised equations are named regularised equations. When we have solved the regularised problem, we direct the factor preceding the small additional term to zero, and the term entered disappears. If, in doing so, the limit of the smooth solution of the regularised equations exists for all $t$, this limit is called a generalised solution of the problem. To be sure, the generalised solution obtained in such a way in general is not smooth. The shortcoming of the approach is that it is possible to get various generalised solutions for the same initial problem, by using various regularizators. Therefore, a correct choice of the regularizator is of great importance. The choice is determined by not only some mathematical means, but also for physical reasons as well.

Let us analyse our problem. We know that $\left|\Theta_{x x x}^{n}\right| \rightarrow \infty$ at the point of wave breaking. Thereby, neglect of $\frac{\beta^{2}}{2}\left(\frac{\gamma-c_{n}^{2}}{\gamma-1} c_{n}^{3}\right.$ $\left.\Theta_{x x x}^{n}\right)$ is inadmissible. The dispersion $\frac{\beta^{2}}{2}\left(\frac{\gamma-c_{n}^{2}}{\gamma-1} c_{n}^{3} \Theta_{x x x}^{n}\right)$ just prevents wave breaking, and it ensures the existence of a smooth solution. Therefore, the small dispersion terms $\frac{\beta^{2}}{2}$. $\left(\frac{c_{n}^{2}}{\gamma-1} c_{n}^{3} \Theta_{x x x}^{n}\right)$ play the role of regularizators. The derivation of equations (2) reveals that the terms $\frac{\beta^{2}}{2}\left(\frac{\gamma-c_{n}^{2}}{\gamma-1} c_{n}^{3} \Theta_{x x x}^{n}\right)$ in (2) originate from $\rho_{0} \partial w / \partial t$ in (1). Hence, one must consider the term $\rho d w / d t$ in (1) as a natural regularisator.

We are going to solve hydrodynamic equations numerically, and the note we now will make is important. We have understood that a classical solution of the quasistatic problem does not exist for all $t$. We account for this fact by the absence of the term $\rho d w / d t$ at this approximation. However, a classical solution for a nonquasistatic problem may be nonexistant as well. For example, a shock wave cannot be interpreted as a classical solution. Thus, in any case some regularisation of the gas dynamics equations (1) is necessary. When we integrate gas dynamics equations numerically, the regularisation is frequently carried out implicitly. So, in computational methods of thorough calculations (Godunov scheme, McCormack scheme, etc.), a "scheme viscosity" is used as a regularizator. We elucidate what we call a "scheme viscosity". Any difference method always gives some consequences of finiteness of grid steps; these are socalled numerical dissipation and numerical dispersion. These numerical effects are parasitic ones. But, at the same time, they play the role of regularizators for a nonlinear problem, ensuring solution existence and uniqueness. These vanishing computational effects are necessary. However, the problem under consideration is unique. In the problem, the competition of various regularizators is possible: the effects of a numerical dissipation and numerical dispersion against the term $\rho d w / d t$. Various regularizators lead to various solutions. To be sure, in a numerical method for nonlinear internal waves, the term $\rho d w / d t$ should take a role as a main regularizator for internal waves, but the effects of a numerical dispersion 
and numerical dissipation should "concede". It is difficult to ensure realisation of this requirement because usually the term $\rho d w / d t$ is very small, while both the numerical dispersion and numerical dissipation are ineradicable due to the finiteness of difference grid steps.

Now we understand the problem specifically, and we are able to formulate some research purposes:

1. To construct a computational model of propagation of nonlinear internal gravity waves in a stratified gas.

2. To investigate a quasistatic limit.

3. To study experimentally the influence of various regularizators on the solution behaviour.

4. To validate the numerical model quality with the help of some special tests based upon the analytical model.

5. To investigate numerically the disintegration of internal gravity waves into solitons.

6. To make use of obtained results to explain some physical phenomena.

The order of these topics will differ from the sectional order for the sake of convenience.

\section{Numerical simulation of nonlinear wave propagation}

\subsection{The numerical model}

Penencko (1985), Peckelis (1988), Tapp and White (1976) and Klemp (1978) constructed nonquasistatic nonlinear models of atmospheric processes. In view of the complexity of the considered equations, some methods of decomposition were widely used to solve them. The models created were not specially aimed at the study of nonlinear internal waves. They were not tested with this class of solutions. Therefore, without additional investigation, it is difficult to tell which method is better for solving the problem.

Let us note some delicacies of numerical integration of gas dynamics equations which originate from the specificity of the problem under consideration.

a) We are interested in internal gravity waves, but acoustic waves exist in a compressible fluid as well. Acoustic waves are excited due to nonlinear effects, as well as through the approximations in numerical methods. Acoustic waves are capable of generating significant errors at numerical simulations, even if the wave amplitudes are small ones. In reality, the approximation error of any numerical method is expressed through the simulation of some higher derivatives of the solution. The acoustic wave frequencies are high and, respectively, the higher derivatives are great. We amplify the previous statement: we are interested in periods that are longer than the internal gravity wave quasiperiods. The periods are huge in comparison to the acoustic wave quasiperiods. The numerical simulation errors have the possibility of accumulating for very long time. Eventually, the numerical solution can essentially differ from the exact one, even if the numerical method is stable. Thereby, we can only hope for some special numerical methods in which the errors associated with acoustic waves are not accumulated.

b) It follows from the analytical theory that, at greater time periods, the solution behaviour is strongly dependent on the ratio between the dispersion term $\rho \partial w / \partial t$ (product of the density and vertical acceleration of fluid particles) and nonlinear terms. All the terms mentioned are small for the problem under consideration. Therefore, with the intension of investigating nonlinear effects, we should make the simulation errors even smaller.

c) An artificial or numerical dissipation is always used to stabilize the numerical simulation of nonlinear gas dynamics. A vanishing numerical dissipation is necessary in order to regularise an acoustic mode of the solution. The specificity of the problem under consideration is that the numerical dissipation can render a huge influence on internal gravity waves. Let us consider a model example. By adding a small artificial dissipation $v B^{n} \Theta_{x x x}^{n}$ into a $\mathrm{KdV}$ equation we convert this equation into a $\mathrm{KdV}$-Burgers equation:

$$
\begin{aligned}
\Theta_{t}^{n}+ & c_{n} \Theta_{x}^{n}+\sigma a^{n} \Theta^{n} \Theta_{x}^{n}+\beta^{2} b^{n} \Theta_{x x x}^{n} \\
& +v B^{n} \Theta_{x x}^{n}=0
\end{aligned}
$$

The dispersion term in (6) is about $\beta^{2} b^{n} A \lambda^{-3}$, and the dissipation term is of order of $\nu B^{n} A \lambda^{-2} ; A$ is the wave amplitude and $\lambda$ is the wavelength. We see that, for sufficiently long waves, the dissipation term is much greater than the dispersion term because $\lambda^{-3}$ vanishes faster than $\lambda^{-2}$ at $\lambda \rightarrow \infty$. When $\nu B^{n} \Theta_{x x}^{n}$ is greater than $\beta^{2} b^{n} \Theta_{x x x}^{n}$, the disintegration into solitons will not take place. In this case the dissipation term $v B^{n} \Theta_{x x}^{n}$ plays the role of a regularizator and some waves such as a shock wave will be generated instead of disintegration into solitons. We take only an interest in long internal waves and there is a real danger in obtaining an erroneous outcome. Therefore, the numerical dissipation is only allowed if the one is brought in some special way so that it does not influence the internal gravity waves.

Careful research of various numerical schemes in the aspects listed is fulfilled in Kshevetskii et al. (1990) and Kshevetskii (1995). The research includes a mathematical proof of a convergence theorem (in a linear approximation) as well as some special investigations of the obstacles listed. It was shown that all numerical schemes of the first order of accuracy (explicit and implicit) are usable to simulate internal wave propagation only if the time step is much less than 3 sec. Otherwise, finiteness of the time step essentially influences the numerical solution obtained. The last undesirable effect originates from the nonlinearity of the equation in some special way (see a). 
Among the numerical methods of the second order of accuracy, some numerical schemes that are more suitable are present. However, it was shown (Kshevetskii, 1995) that the time step $\tau$ as well the step $h_{1}$ along a horizontal coordinate must satisfy inequalities:

$\tau \ll 4 \sqrt{\frac{H}{g}}, \quad h_{1} \ll \sqrt{2} H$.

Otherwise, numerical effects of dissipation or dispersion will play the role of a regularizator of the nonlinear problem instead of the term $\rho d w / d t$. This will considerably change the behaviour of the nonlinear internal gravity waves and is inadmissible. We note the uncommonness of this statement. Such subtle questions were not formerly considered in the literature.

Careful numerical experiments have revealed that even computer roundings essentially influence the numerical solution. The effects of computer roundings are well known, but they seldom are important in practice. Their present importance can be explained by two circumstances. First, we solve the problem for large intervals of heights $(0-100 \mathrm{~km})$, while the atmospheric gas density is exponentially decreasing with height. Secondly, we are interested in the behaviour of atmospheric parameters for large time spans. The influence of rounding errors can be imperceptible during one hour. Nevertheless, the effect becomes significant for large time spans, because small rounding errors are accumulated over time. To avoid the rounding error effect, the calculations were organised in a special way. At each integrating step, the wave components in relation to the background pressure, density, and temperature were calculated as preliminary ones and then the pressure, density and temperature were calculated by means of adding the wave components to the background values.

Due to the research, some special numerical models were constructed. In a linear quasistatic approximation, the numerical scheme developed is analogous to the scheme suggested by Yudin and Gavrilov (1985), but is slightly advanced. Some special grid of the type "cross" (Samarskiy and Popov, 1980) has been used in order that the difference equations are more exact. In addition, the grid "cross" is convenient because it easily allows the inspecting realisation of conservation laws.

The numerical scheme constructed is very similar to the two-step Lax-Wendroff scheme (Richtmyer and Morton, 1967). Therefore, it is convenient to describe the algorithm peculiarities by starting with this known scheme. Lax and Wendroff considered the hydrodynamic conservation laws

$r_{t}+(q(r))_{x}+(s(r))_{z}=0$.

Here $r$ is a vector function, whose components are the density, momentum density and energy density. Lax and Wendroff have approximated these conservation laws as follows:

$\frac{r_{i, k}^{j+1}-r_{i, k}^{j}}{\tau}+\frac{q_{i+\frac{1}{2}, k}^{j+\frac{1}{2}}(r)-q_{i-\frac{1}{2}, k}^{j+\frac{1}{2}}(r)}{h_{1}}$

$$
+\frac{s_{i, k+\frac{1}{2}}^{j+\frac{1}{2}}(r)-s_{i, k-\frac{1}{2}}^{j+\frac{1}{2}}(r)}{h_{2}}=0 .
$$

Here $\tau$ is a time step, $h_{1}, h_{2}$ are spatial steps that are horizontal and vertical. A deviation from the usual gas dynamics equations considered by Lax and Wendroff, we take into consideration the gravity in the equation for momentum density. With the point of view of numerical methods, this deviation does not entail any serious difficulties.

More importantly, the mathematical difference of our scheme in comparison to the classical one is that an implicit approximation is used at the first half-step:

$$
\begin{gathered}
2 \frac{r_{i, k}^{j+\frac{1}{2}}-r_{i, k}^{j}}{\tau}+\frac{q_{i+\frac{1}{2}, k}^{j+\frac{1}{2}}(r)-q_{i-\frac{1}{2}, k}^{j+\frac{1}{2}}(r)}{h_{1}} \\
+\frac{s_{i, k+\frac{1}{2}}^{j+\frac{1}{2}}(r)-s_{i, k-\frac{1}{2}}^{j+\frac{1}{2}}(r)}{h_{2}}=0 .
\end{gathered}
$$

It certainly complicates the computations. However, this peculiarity of the scheme is very important. (The errors originating from the acoustic waves are not only accumulated in the difference schemes of such a structure.)

The numerical scheme constructed has one additional difference from the classical Lax-Wendroff scheme. One can use the numerical scheme to simulate processes in which simultaneously, both internal gravity waves and acoustic waves take part. We already noted that a vanishing dissipation is necessary as a regularizator for acoustic waves. However, this numerical dissipation must not influence internal gravity waves. It is reasonable to enter the numerical dissipation for acoustic waves by using "downstream differences" for approximating spatial derivatives in $d(\rho w) / d t$. This approximation introduces some additional effects of a vanishing nonlinear dissipation. However, the resulting numerical dissipation renders minimum influence on internal gravity waves because the term $d(\rho w) / d t$ is very small for internal gravity waves. The numerical scheme as it stands contains two various selectively operating regularizators.

We note especially that no additional addends are intoduced into the equations. All useful qualities of the method are achieved in conventional receptions, but at the expense of successful selection of difference approximations and use of sufficiently small steps of the difference grid. Some small amendments improving dispersion relation and diminishing scheme sensitivity to the choice of grid steps were made as well.

The opinion proposed on numerical methods is not a substitute for conventional approaches in consideration of difference methods; it only supplements them. The research of stability and convergence has been carried out, but they are not considered here. We have considered and compared among themselves only stable numerical methods. 


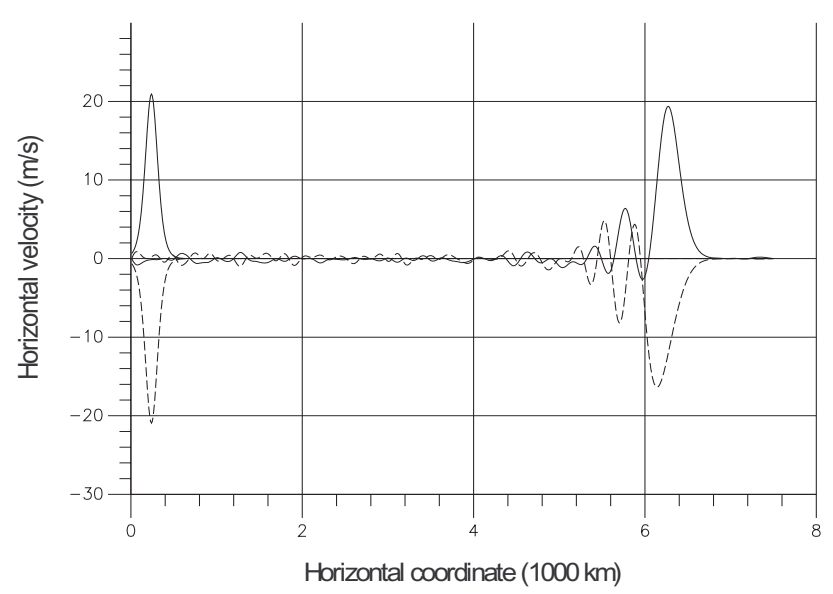

Fig. 1. Propagation of a soliton. The height is equal $98 \mathrm{~km}$. Propagation of the wave having an opposite sign at zero time is represented for comparison by a dotted line.

\subsection{Comparison of analytical and numerical models}

We shall show here some outcomes of numerical simulation of nonlinear internal waves and we shall compare them with the analytical model. The background parameters were selected for the Earth's atmosphere conditions $(100 \mathrm{~km})$. For simplification, the scale height $H$ is a constant $(H=8 \mathrm{~km})$, dissipation effects are not taken into account. The boundary conditions of a rigid cover are imposed at the Earth's surface and at the height $100 \mathrm{~km}$.

The analytical model shows that wave modes behave quasi-independently, if the wave amplitude is sufficiently small. Therefore, the example of a one-mode wave is sufficient to verify analytical outcomes. At that, it reveals the essence of nonlinear processes in the best way. The first mode of internal waves was selected for tests. In the analytical model, for simplification, we have neglected the nonlinear interaction of this mode with others. Within the framework of the analytical model, the considered wave propagates strictly horizontally. To be sure, the numerical hydrodynamic model takes into account all nonlinear effects.

In the analytical model, the hydrodynamic functions are expressed through $\Theta^{n}$ as follows:

$$
\begin{aligned}
& u(x, z, t)=\Theta^{n}(x, t) \\
& \cdot\left(A_{1} \sin \left(k_{z} z\right)+B_{1} \cos \left(k_{z} z\right)\right) \exp \left(\frac{z}{2 H}\right), \\
& w(x, z, t)=-c_{n} \frac{\partial}{\partial x} \Theta^{n}(x, t) \sin \left(k_{z} z\right) \exp \left(\frac{z}{2 H}\right), \\
& \Delta P(x, z, t)=g H \Theta^{n}(x, t) \\
& \cdot\left(A_{2} \sin \left(k_{z} z\right)+B_{2} \cos \left(k_{z} z\right)\right) \exp \left(-\frac{z}{2 H}\right) \rho_{00}, \\
& \Delta \rho(x, z, t)=\Theta^{n}(x, t) \\
& \cdot\left(A_{3} \sin \left(k_{z} z\right)+B_{3} \cos \left(k_{z} z\right)\right) \exp \left(-\frac{z}{2 H}\right) \rho_{00}, \\
& A_{1}=\frac{c_{n} g(2-\gamma)}{2\left(c_{n}^{2}-\gamma g H\right)}, \quad A_{2}=\frac{c_{n}^{2}(\gamma-2)}{2 H\left(\gamma g H-c_{n}^{2}\right)},
\end{aligned}
$$

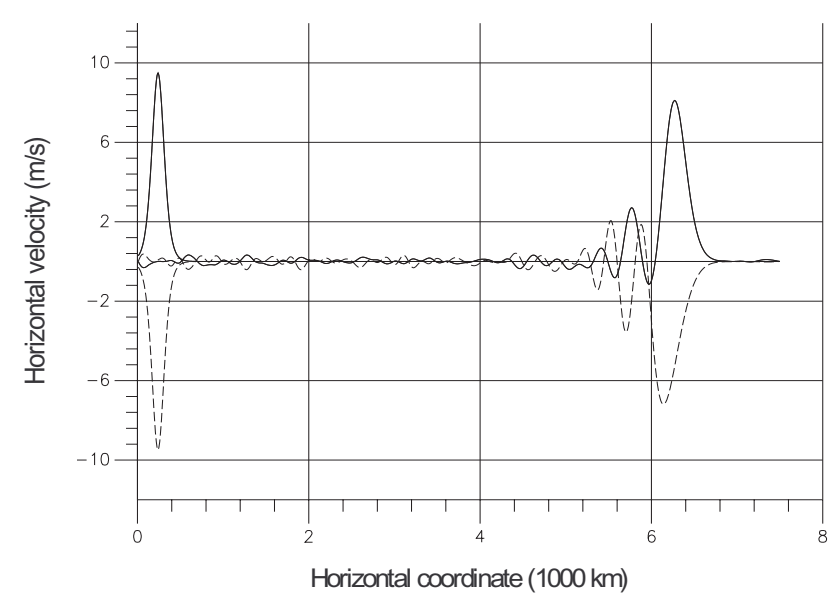

Fig. 2. Propagation of a soliton. The height is equal $82 \mathrm{~km}$. Propagation of the wave having an opposite sign at zero time is shown for comparison by a dotted line.

$A_{3}=\frac{c_{n}^{2}-2 g H(\gamma-1)}{2 H\left(c_{n}^{2}-\gamma g H\right)}, \quad B_{1}=\frac{\gamma g H k_{z} c_{n}}{\gamma g H-c_{n}^{2}}$,

$B_{2}=\frac{c_{n}^{2} \gamma k_{z}}{\gamma g H-c_{n}^{2}}, \quad B_{3}=\frac{c_{n}^{2} k_{z}}{-c_{n}^{2}+\gamma g H}$,

$c_{n}^{2}=\frac{4(\gamma-1) g H}{\gamma\left(1+4 k_{z}^{2} H^{2}\right)}$.

Here $u$ and $w$ are the horizontal and vertical velocities; $\Delta P$, $\Delta \rho$ are the wave components to the background pressure $P_{0}(z)=\rho_{0}(z) g H$ and the background density $\rho_{0}(z)=$ $\rho_{00} \exp (-z / H) ; \rho_{00}$ is the density at the Earth's surface; $k_{z}=n \pi /$ (waveguideheight) is a vertical component of the wave vector. For the first mode, $n=1$.

The Korteweg-de Vries equation (4) for the mode with $n=$ 1 , in dimensional variables, looks like

$$
\begin{aligned}
\Theta_{t}^{n}+ & 1.03 \sqrt{g H} \Theta_{x}^{n}-212 \sqrt{\frac{g}{H}} \Theta^{n} \Theta_{x}^{n} \\
+ & 0.478 H^{2} \sqrt{g H} \Theta_{x x x}^{n}=0 .
\end{aligned}
$$

In order to allow for no errors in the test example, all calculations were carried out with the help of the Derive program. If the initial condition of the Korteweg-de Vries equation (8) is as follows

$$
\Theta^{n}(x, 0)=-\frac{6 N(N+1) 0.478 H^{3}}{212 L^{2}} \cosh ^{-2}\left(\frac{x-x_{0}}{L}\right),
$$

then exactly $N$ solitons will be generated at $t \rightarrow \infty$ (Lamb, 1980). We will use this fact for our tests.

The propagation of one soliton simulated by direct numerical integration of hydrodynamic equations is shown in Figs. 1,2. The wave disturbance after 313 minutes is shown by a solid line on the right. For a comparison, the similar wave, but of an opposite sign at the initial instant, is represented in the same figures by a dashed line. This wave dampens slowly over the course of time, while the soliton keeps the amplitude essentially better. The numerical outcomes certainly agree 


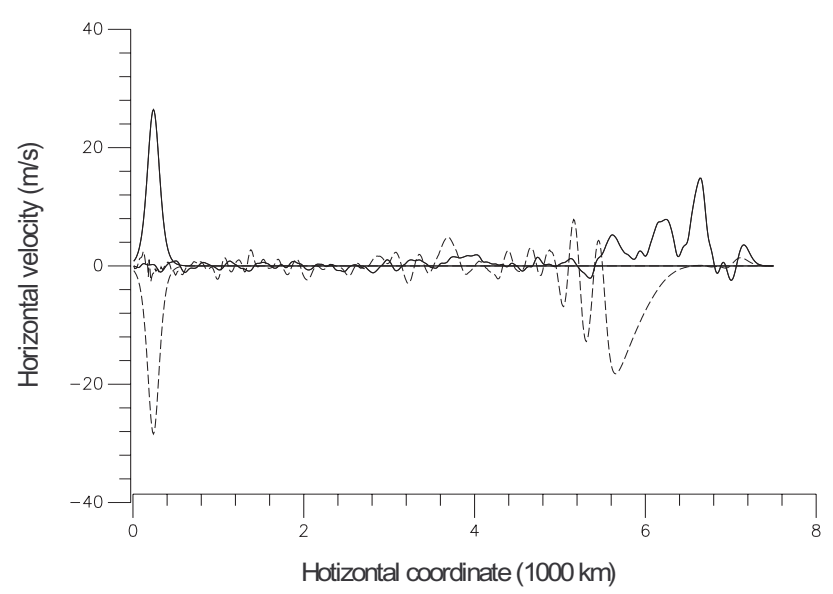

Fig. 3. Propagation of a 4-soliton wave. The height is equal 66 $\mathrm{km}$. For comparison, a dotted line displays propagation of the wave having an opposite sign at zero time.

with the analytical ones. Nevertheless, some parasitic smallamplitude waves are observed. Probably, one can explain it by the fact that the analytical soliton solution is only an approximate one. The nonlinear effects are feeble, but the wave amplitude is also small. Unfortunately, now we know of no analytical solutions for internal waves of considerable amplitudes.

The disintegration of a nonlinear internal wave into solitons is a very bright physical process. The propagation of a disintegrating 4-soliton wave is shown in Figs. 3, 4. The initial conditions had been taken so that precisely four solitons were eventually generated (that is, $N=4$ in (9)). The initial conditions are shown on the left by a solid line and the wave after 313 minutes is represented on the right. We see that three solitons were generated at the instant shown in the figures. The outcomes of numerical experiments are in an acceptable consent with the outcomes of analytical research (Kshevetskii and Leble, 1985, 1988; Kshevetskii, 1998).

When one keeps in mind the typical atmospheric waves, then the wave amplitude may be considered as a bit excessive. The amplitude of the horizontal velocity is equal to $150 \mathrm{~m} / \mathrm{s}$ at the height $98 \mathrm{~km}$, while actually the wind at less than $150 \mathrm{~m} / \mathrm{s}$ is more probable for these heights. However, in our test example we have not taken into consideration the dependence of $H$ on $z$. This dependence would lead to a partial wave reflection from the mesopause region and, consequently, the actual wave amplitude would be less. We could use the 4-soliton wave of a smaller amplitude by taking the parameter $L$ smaller. This parameter is a free one, and we have selected it to facilitate the numerical simulation. It is more difficult technically to carry out numerical experiments with waves of smaller amplitudes. Supposing we have used the initial conditions for the 4-soliton wave with the amplitude smaller by $p$ times, then the scale $L$ of the 4 -soliton wave would be larger by $\sqrt{p}$ times. The time of disintegration of such a wave would be longer by $p \sqrt{p}$ times. It complicates the research because the danger of numerical error

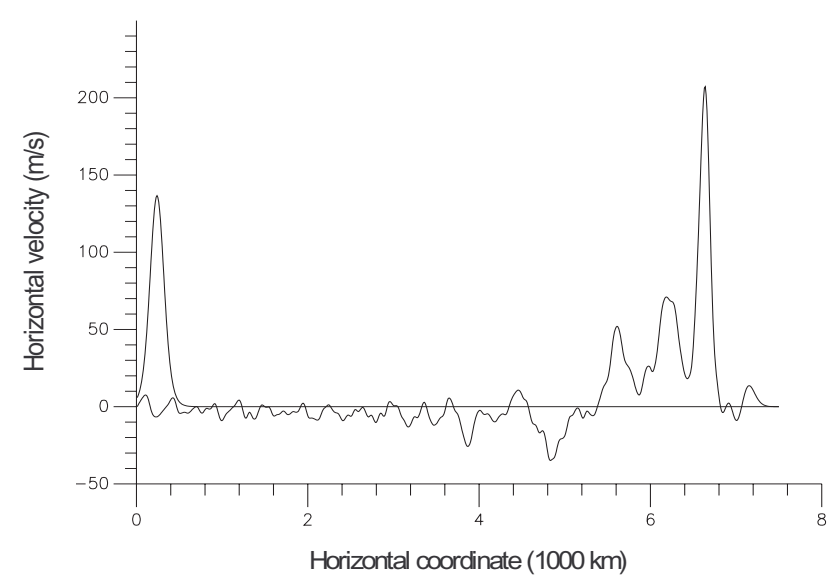

Fig. 4. Propagation of a 4-soliton wave. The height is equal $98 \mathrm{~km}$.

accumulation is increasing. At the same time, such updating of the initial conditions can provide no new outcomes. The less the wave amplitude is, the more precisely the analytical theory works. It is evident.

The wave disintegration is a corollary of nonlinearity. To demonstrate it, the propagation of the wave having an opposite sign in initial conditions is shown by a dashed line in Fig. 3. We see that wave disintegration does not happen in this case. It confirms the outcome obtained analytically. The steepness of this wave increases not at the wave front, but at the back. This perfectly agrees with the analytical outcomes as well.

Notwithstanding coincidence of many details, the analytical formulas are somewhat rough. They display the vertical wave structure inaccurately. The amplitude of the numerical solution grows with height faster than the amplitude of the analytical one. Probably, we would achieve better coincidence of outcomes if we had taken into consideration, in the analytical formulas, the nonlinear effects of induced perturbation of other modes by our mode, at the expense of nonlinear effects (Kshevetskii and Leble, 1985, 1988).

It is possible to see in consideration of the analytical formulas that each soliton of the first wave mode is a vortex. Therefore, one can interpret the disintegration into solitons as a disintegration of an initial vortex into more small-scale vortexes.

A characteristic wave tail similar to turbulence is left behind the principal wave. It fails to explain this wave tail by numerical effects, because the grid steps are much less than the typical scale of oscillations in the tail. This wave turbulent tail is not the tail described by a non-soliton solution of a $\mathrm{KdV}$ equation, because the wave tail propagates too slowly. It is known that the internal waves of short vertical scales have small propagation velocities. The wave tail consists of such short waves along the vertical. The reasons for the wave tail generation are not quite understood; this effect is not yet investigated.

An indented nonsmooth horizontal structure of the wave tail is of interest. It is not simply wave oscillations. These 


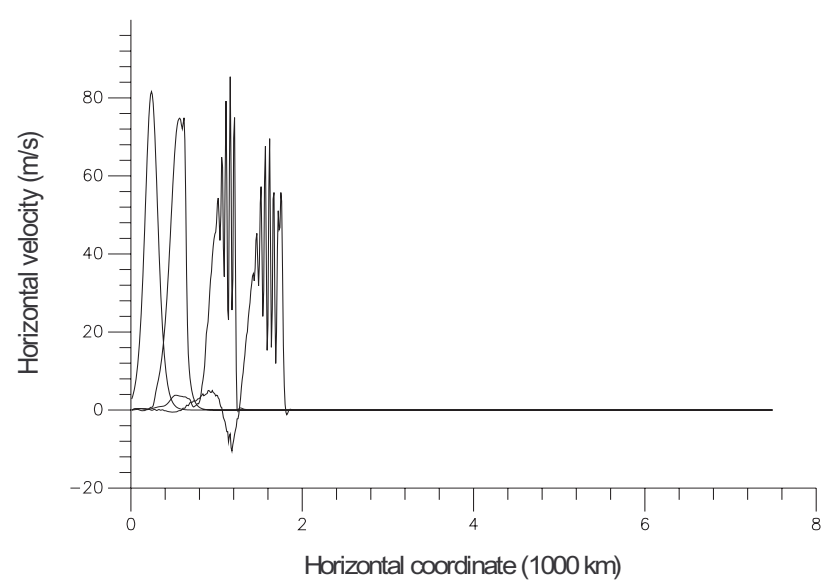

Fig. 5. Wave propagation corresponding to the same initial conditions as in 4-soliton case, but in a quasistatic model. The height is equal $84 \mathrm{~km}$. No regularization is utilized. The wave is wrecked.

are nonsmooth or almost nonsmooth waves. The wave tail consists of small-scale vortices. It is possible to put forward the following hypothesis explaining the tail irregularity. The dispersion constants $\frac{\beta^{2}}{2}\left(\frac{\gamma-c_{n}^{2}}{\gamma-1} c_{n}^{3}\right)$ are very small for short waves along the vertical. The wave tail consists of such waves. The constants are small of the order $O\left(\beta^{2} / n^{3}\right), n$ is the mode number, $n$ is large because short waves along the vertical are considered. The less the values of the dispersion constants are, the shorter the solitons generated through disintegration. That is, if one accepts that the tail consists of solitons of higher modes, then the solitons in the tail should be of very small horizontal scales. We concluded that short waves along the vertical have to become short along the horizontal, at the expense of wave disintegration. It takes place in Figs. 3, 4 actually: the generation of some nonstationary structure consisting of a number of small vortices remaining behind the head wave.

The quasistatic approach is very popular in atmospheric models because essentially it simplifies simulation. However, the analytical theory reveals that the solution of a nonlinear quasistatic problem can be nonexistant for some $t$. To verify this conclusion, some special numerical experiments were carried out. The term of vertical acceleration in hydrodynamic equations was discarded, and then the equations were solved numerically. In Fig. 5, the behaviour of the same wave as in the 4-soliton case is shown, but within the framework of a quasistatic approach. Some time later, during "normal evolution", the wave collapses and a simulation emergency stop arises.

Usually one achieves correctness of such nonlinear problems by means of adding some vanishing artificial (or numerical) dissipation. Under our prognoses, such a method of problem regularisation should result in the wave like a shock wave. We cannot bypass this interesting and intriguing subject. The outcome of a numerical simulation of nonlinear internal waves with the quasistatic model regularised

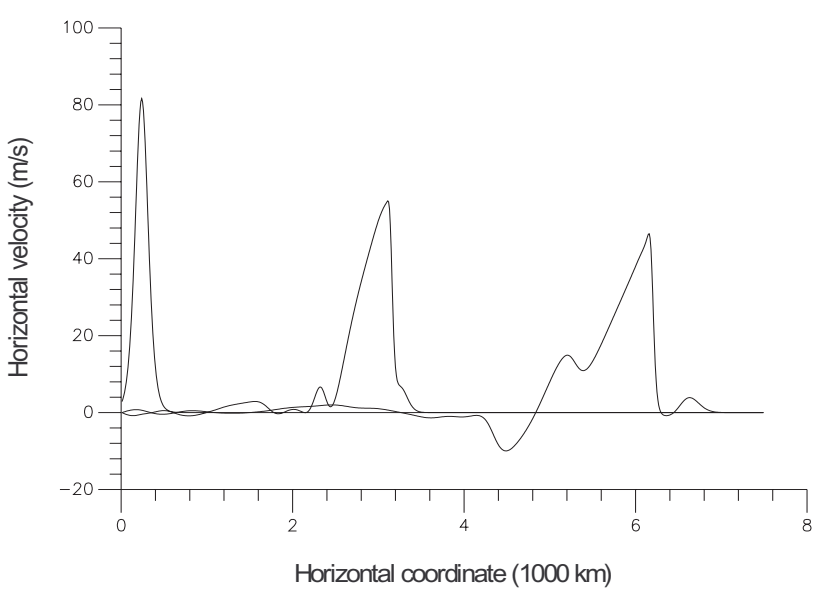

Fig. 6. Propagation of the wave corresponding to the same initial conditions as in 4-soliton case, but in a quasistatic model. A vanishing artificial dissipation was used as a regularizator. A "shock wave" is formed. The height is equal $84 \mathrm{~km}$.

with a vanishing artificial viscosity is shown in Fig. 6. The initial conditions are the same as in the case with four solitons. The vanishing dissipation has completely stabilised the wave behaviour and the wave behaves similarly to a shock wave. However, the trajectories of liquid particles are different ones. A shock wave is a wave of compression and the particles move perpendicularly to the shock wave wavefront. In the present case, we observe a propagating vortex and, at the wave front, the particles move in parallel to the wavefront. In the course of time, the vortex is strongly deformed on the front. Disintegration of this vortex into small-scale vortexes does not take place within the approach under consideration.

The solution obtained is independent of the artificial viscosity factor and one can consider it as one of several possible generalised solutions of a quasistatic problem. The hydrodynamic equations, in their initial form, are very difficult for the intuitive understanding of the nature of this generalised solution. Equations (5) allow one to explain perfectly "mathematical effects". So, the generalised solution obtained is nothing else but a generalised solution of the set of equations (5), which is obtained from the solution of the set of equations

$\Theta_{t}^{n}+c_{n} \Theta_{x}^{n}+\frac{\sigma}{2} \sum_{m, l} F_{l, m}^{n} \Theta^{l} \Theta_{x}^{m}+v \sum_{m} K_{m}^{n} \Theta_{x x}^{m}=0$,

$K_{n}^{n}<0$,

by means of passage to the limit $v \rightarrow 0$. We can easily write down an approximate analytical solution of this set of equations. The approximate solution looks like (3), (4), but the Korteweg-de Vries equation (4) must be replaced by the Burgers equation

$\Theta_{0 t}^{n}+c_{l} \Theta_{0 x}^{n}+\frac{\sigma}{2} F_{n n}^{n} \Theta_{0}^{n} \Theta_{0 x}^{n}+v K_{n}^{n} \Theta_{0 x x}^{n}=0$.

It is well known that if $F_{n n}^{n} \Theta^{n}(x, 0) \geq 0$ and also if $\Theta^{n}(x, 0)$ is a compactly supported disturbance, then the solution of 
a Burgers equation provides the wave like a shock wave at $v \rightarrow 0$. We observe such a wave in the numerical experiment conducted.

From the mathematical point of view, the generalised solution obtained is faultless. However, a qualitative difference from the solution of a nonquasistatic problem is obvious. Another regularizator (artificial viscosity instead of dispersion) has generated an other generalised solution of the nonlinear problem. If we have not particularly analysed the subtle situation with equation regularisation and if we have not undertaken some special measures to guard against the numerical effects, then we would easily obtain such a "solution" even with a nonquasistatic model. For example, the investigation has shown that an implicit scheme of the first order of accuracy inevitably produces the same outcome, even if only the incredibly severe constraint $\tau \ll 3 \sec$ (Kshevetskii, 1995) is not satisfied.

Let us discuss briefly the applicability of obtained results to the actual atmospheric waves. In the investigated model, a temperature stratification and dissipation is not taken into account. The dissipation effects are negligible in the real atmosphere below $100 \mathrm{~km}$, but exponentially increase with height, and are very significant above $250 \mathrm{~km}$ (Gossard and Hook, 1978; Dikiy, 1969). The analytical model and outcomes obtained reveal that the modes behave quasi-independently at sufficiently small amplitudes. Using several of these quasiindependent modes, we could have even simulated vertical wave propagation, down to the upper boundary. Analogy to the Fourier method is relevant here. Therefore, it is hardly probable that the upper boundary condition or dissipation being increased with height, can considerably influence the results obtained. The investigated nonlinear effects should evince one's force irrespectively of the boundary conditions. In particular, the nonlinear disintegration of internal waves into solitary waves of smaller scales must take place in the real atmosphere.

In the paper, nonlinear waves in an incompressible fluid were not particularly studied, but these waves correspond to the limit $\gamma \rightarrow \infty$. The approximation of an incompressible fluid is usually utilized to describe ocean waves. Therefore, the author hopes that the results obtained can be useful for understanding the ocean waves as well. Furthermore, now the KdV model is actively used in oceanology for the study of internal waves and interpretation of observations. The KdV model seems to be a convenient one to describe ocean waves because ocean waves propagate within a natural wave-guide. When considering atmospheric waves, we have applied the single-mode analytical $\mathrm{KdV}$ model for qualitative understanding of atmospheric nonlinear processes. We do not lay claim to a quantitative description of atmospheric nonlinear waves with such a simple model. In consideration of ocean waves, the elementary analytical model can give quite a good quantitative consent.

\section{The KdV model and internal wave mixing}

In oceanology, it is known that a smooth internal gravity wave can suddenly break up, generating a spot, inside which a turbulent fluid intermixing takes place. This effect is frequently named "internal wave mixing". We now consider the internal mixing from the point of view of equations (2), and we shall analyse the conditions when the effect takes place.

Formula (3) gives an approximate solution of (2). Uncoupled KdV equations (4) lie in the basis of (3). Let us take initial conditions analogous to (9)

$\Theta_{0}^{n}(x, 0)=-\alpha_{n} \cosh ^{-2}\left(\frac{x-x_{0}}{L_{n}}\right)$,

where

$\alpha_{n}=-\frac{6 N(N+1) H^{3} \beta^{2} \frac{\gamma-c_{n}^{2}}{\gamma-1} c_{n}^{3}}{L_{n}^{2} \sigma F_{n n}^{n}}$.

The formulas (11), (12) can be interpreted as follows: if initial conditions look like (11), then the amount $N$ of solitons to be generated may be derived from (12). It is clear that, for fixed $\alpha_{n}$ and $L_{n}$, the less $c_{n}$ the more solitons will be generated. If $c_{n} \rightarrow 0$, then $N \rightarrow \infty$. The integrals $\int_{-\infty}^{+\infty} \Theta^{n} d x$ are conservative values for equation (4). Therefore, if $N \rightarrow \infty$, then the soliton scales tend to zero. That is, if $c_{n}$ is very small, then a huge number of extremely small-scale solitons will be generated. Each soliton formed gives a vortex. Therefore, the physical phenomenon under study is the same, because a smooth initial wave disintegrates into a huge number of small vortexes. The constants $c_{n}$ are of the order $h /(n \pi)$, where $n$ is the wave mode number and $h$ is the wave-guide depth. Consequently, the limit $c_{n} \rightarrow 0$ is equivalent to the limit $n \rightarrow \infty$. We see that only short waves along the vertical, that is, such that $H / l_{z} \gg 1$, can disintegrate into many small-scale solitons. The symbol $l_{z}$ designates a typical vertical scale of the wave. Due to the short vertical wave scales, the processes happening far from wave-guide boundaries, in the body of the fluid, weakly depend on the boundary conditions.

McEwan (1983) investigated experimentally the effect of "internal wave mixing". In Fig. 7, a common picture of the considered phenomenon is shown; it is borrowed from McEwan (1983).

According to the estimates made above, the effect takes place only for short waves along the vertical. Let us check whether this requirement is satisfied at the conditions of McEwan's experiment (1983). In McEwan's experiment, $\left(g \rho^{-1} d \rho / d z\right)^{1 / 2} \approx 1.23 c^{-1}$, and the tank depth $l$ was equal to $25 \mathrm{~cm}$. Hence, $H \approx 6 \mathrm{~m}$, and $k_{z} \geq \pi / l \approx 4 \mathrm{~m}^{-1}$. We see that $k_{z} H>20$ in McEwan's experiment. Doubtlessly, McEwan dealt with short waves along the vertical.

We consider nonlinear effects for modes with large $n$. As noted above, the effect of the interaction of modes $n$ and $m$ is proportionate to $\left(c_{n}-c_{m}\right)^{-1}$. When $m=n+1$ and at $n \rightarrow \infty$, the fraction denominator $\left(c_{n}-c_{n+1}\right)$ is a small of the order $O\left(n^{-2}\right)$. We have neglected above the interactions 


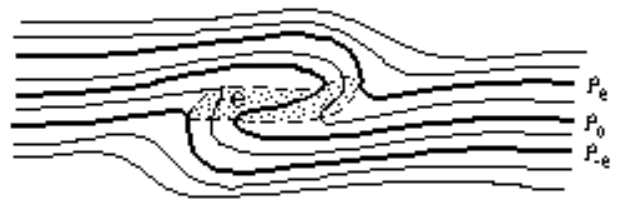

[a]

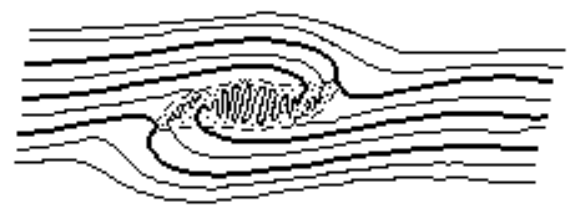

[b]

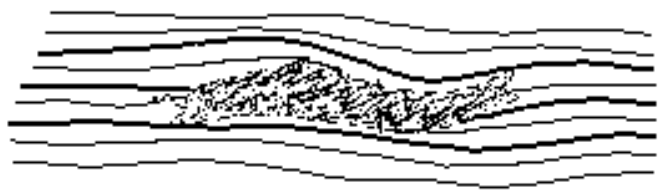

[c]

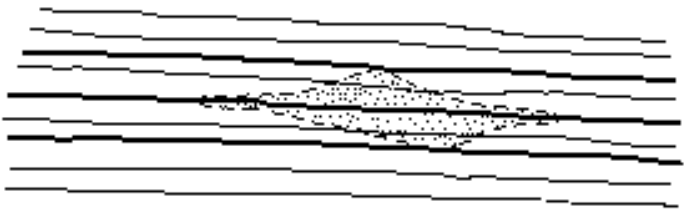

[d]

Fig. 7. Idealisation of a mixing event in a continuous stratification. (a) Overturning. (b) Development of interleaving microstructure. (c) Static stability is restored, but microstructure is preserved. (d) Gravitation to equilibrium has changed the surrounding density between extremum isopycnals.

of various modes and, in order that this simplification will be correct, we have to require $\alpha_{n} \sim c_{n}^{2} / g \sim n^{-2}$. Fortunately, the dispersion coefficients $\frac{\beta^{2}}{2}\left(\frac{\gamma-c_{n}^{2}}{\gamma-1} c_{n}^{3}\right)$ vanish very quickly at $n \rightarrow \infty$, as $c_{n}^{3} \sim(\sqrt{g / H}(h / n))^{3} \rightarrow 0$. It rescues our idea: the amount $N$ of solitons generated is determined only by a relationship between the nonlinearity and the dispersion, and will be huge, because the nonlinearity considerably surpasses the dispersion. One can consider the idea as a "mathematical explaining" of internal wave mixing. Unfortunately, the considered model does not lead to simple and convenient working formulas. We can only suggest qualitative dependencies of the scale $l_{x}$ and quantity $N$ of generated solitons on parameters of the disintegrating wave:

$l_{x} \sim \sqrt{\frac{\left(l_{z}\right)^{3}}{A}}, \quad N \sim \sqrt{\frac{A L^{2}}{l_{z}^{3}}}$.

Here $l_{z}$ is a vertical scale of the broken up wave; $A$ is a mean amplitude of displacement of the fluid particles, $L$ is a horizontal scale of the broken up wave.

We can supplement McEwan's outcomes with some qualitative notes:

- If $H \rightarrow \infty$ (or, this is the same, if $g \rightarrow 0$ ), then $c_{n} \rightarrow 0$, and the effect of wave disintegration can take place as well. It depends on initial conditions. When $H \rightarrow \infty$, the internal gravity waves turn into some stationary flow in homogeneous liquid. Hence, a fluid stratification in itself is not a reason for wave disintegration. The stratification only provides a bright observation of the effect.

- We try to formulate some abstract mathematical models of the phenomenon. The phenomenon exists because hydrodynamic equations have the following structure

$\widehat{N}\left(\frac{\partial}{\partial t}, \frac{\partial}{\partial x}\right) \theta(x, t)=\varepsilon \widehat{D} \theta(x, t)$.

Here $\widehat{N}$ is a nonlinear operator, such that a smooth solution of the equation $\widehat{N}(\partial / \partial t, \partial / \partial x) \theta(x, t)=0$ does not exist for some $t=t_{1} ; \varepsilon \ll 1$; and $\varepsilon \widehat{D} \theta(x, t)$ is a small dispersion term which plays the role of a regularizator. The small dispersion term ensures the existence of a differentiable solution of equation (13), but this differentiable solution is quickly varying. The less $\varepsilon$ is, the faster the solution varies. We have written spatially one-dimensional equations, but it is not important. All remaining conditions are the important ones.

- We have described above some difficulties of numerical integration of hydrodynamic equations. These difficulties in many respects follow from the fact that the basic equations have the structure (13). We see that the addition of some small dispersion or dissipation terms into the equations can change the wave behaviour considerably. This sensitivity in relationship to terms containing higher derivatives takes place because a smooth solution of the equation $\widehat{N}(\partial / \partial t, \partial / \partial x) \theta(x, t)=0$ does not exist for some $t=t_{1}$. We deal with certain specific cases of a nonlinear system instability.

\section{Conclusions}

1. Hydrostatic and nonhydrostatic numerical hydrodynamic models of nonlinear internal wave propagation are developed. Also, an analytical model is developed and is used to explain nonlinear wave behaviour.

2. The nonlinear disintegration of internal waves into solitary waves of smaller scales is simulated. The comparison of the outcomes of numerical simulation with analytical ones has shown qualitative consent. For example, the quantity of solitons generated is displayed precisely.

3. Numerical experiments have confirmed that a quasistatic approximation leads to gradient catastrophe.

4. Influence of various regularizators on the quasistatic solution is investigated. Numerical experiments have revealed that a nonlinear catastrophe in the quasistatic 
model is prevented with the help of a vanishing artificial or numerical dissipation, but the solution obtained qualitatively differs from the solution of a nonquasistatic problem for large time spans. The small term $\rho \partial w / \partial t$ in nonhydrostatic hydrodynamic equations plays the role of a natural dispersion regularizator, with respect to a quasistatic problem.

5. The analytical model qualitatively explains the effects of internal wave mixing. It is shown that the effect takes place for internal short waves along the vertical, and that stratification is not a reason of the phenomenon. The same nonlinear mechanism acts in a homogeneous liquid, producing extremely small-scale vortexes from some large-scale flows.

\section{Appendix A The derivation of equations for wave modes}

In this appendix the derivation of equations (2) is given. We shall deduce here some equations, more commonly compared with (2), which take into account not only nonlinearity, but also the influence of a weak dissipation on wave propagation. Let principal equations be $2 \mathrm{D}$ gas dynamics equations taking into account the gravity force:

$$
\begin{aligned}
& \frac{\partial \rho}{\partial t}+\rho(\nabla \cdot \boldsymbol{v})=0, \\
& c_{v} \frac{d T}{D t}=-R T(\nabla \cdot \boldsymbol{v})+\frac{k}{\rho} \nabla \cdot(k \nabla T), \\
& \rho \frac{d \boldsymbol{v}}{d t}=-\nabla P-\rho g+\nabla(\eta \nabla \cdot \boldsymbol{v}), \\
& P=\frac{\rho}{\mu} R T,
\end{aligned}
$$

Here $P$ is the pressure; $\rho$ is the density; $T$ is the temperature; $\boldsymbol{v}=\{u, w\}$ is a vector of the gas velocity with projections $u, w$ onto axes $x, z ; c_{v}$ is an isochoric molar thermal capacity of the gas; $\mu$ is a molar weight of the gas. $R$ is the universal gas constant; $\eta$ is a viscosity coefficient of the gas; $k$ is the thermal conductivity. $g$ is the gravity acceleration. The axis $z$ is upward.

The problem under consideration is characterised by small dimensionless parameters:

$$
\begin{aligned}
& \sigma=\frac{\Delta T}{T_{0}}, \quad \beta=\tau \sqrt{g / H}, \\
& \nu(z)=\frac{\eta(z)}{\beta H \rho_{0}(z) \sqrt{g H}}, \\
& \Omega(z)=\frac{k(z) \mu}{c_{v} \beta \rho_{0}(z) H \sqrt{g H}} .
\end{aligned}
$$

Here $\Delta T$ is the amplitude of temperature variation at wave propagation; $\tau$ is the wave quasiperiod; $\rho_{0}(z)$ is the nonperturbed density; $T_{0}$ is the nonperturbed temperature that is assumed be a constant; $H=R T_{0} /(g \mu)$ is the scale height.

After transformation to dimensionless variables

$$
t^{\prime}=\beta \sqrt{\frac{g}{H}} t, \quad x^{\prime}=\frac{\beta x}{H}, \quad z^{\prime}=\frac{z}{H},
$$

$$
\begin{aligned}
\phi^{\prime} & =\frac{T-T_{0}}{T_{0}}, & \psi^{\prime} & =\frac{\rho-\rho_{0}}{\rho_{0}}, \\
u^{\prime} & =\frac{u}{\sigma \sqrt{g H}}, & w^{\prime} & =\frac{w}{\sigma \beta \sqrt{g H}},
\end{aligned}
$$

the basic equations are brought to the form

$$
\begin{aligned}
& \psi_{t^{\prime}}^{\prime}-w^{\prime}+w_{z^{\prime}}^{\prime}+u_{x^{\prime}}^{\prime} \\
& \quad=-\sigma\left(\psi_{x^{\prime}}^{\prime} u^{\prime}+\psi_{z^{\prime}}^{\prime} w^{\prime}+\psi\left(w_{z^{\prime}}^{\prime}+u_{x^{\prime}}^{\prime}\right)\right), \\
& u_{t^{\prime}}^{\prime}+\psi_{x^{\prime}}^{\prime}+\phi_{x^{\prime}}^{\prime}=-\sigma\left(\left(1+\sigma \psi^{\prime}\right)\left(u^{\prime} u_{x^{\prime}}^{\prime}+w^{\prime} u_{z^{\prime}}^{\prime}\right)\right. \\
& \left.\quad+\left(\psi^{\prime} \phi^{\prime}\right)_{x^{\prime}}\right)+v u_{z^{\prime} z^{\prime}}^{\prime}, \\
& \psi_{z^{\prime}}^{\prime}+\phi_{z^{\prime}}^{\prime}+\psi^{\prime}=-\sigma\left(\phi^{\prime} \psi^{\prime}\right)_{z^{\prime}} \\
& \quad-\beta^{2}\left(1+\sigma \psi^{\prime}\right)\left(w_{t^{\prime}}^{\prime}+\sigma\left(u^{\prime} w_{x^{\prime}}^{\prime}+w^{\prime} w_{z^{\prime}}^{\prime}\right)\right), \\
& \phi_{t^{\prime}}^{\prime}+(\gamma-1)\left(u_{x^{\prime}}^{\prime}+w_{z^{\prime}}^{\prime}\right)=-\sigma\left[\phi_{x^{\prime}}^{\prime} u^{\prime}+\phi_{z^{\prime}}^{\prime} w^{\prime}\right. \\
& \left.\quad+(\gamma-1)\left(\phi^{\prime} u_{x^{\prime}}^{\prime}+\phi^{\prime} w_{z^{\prime}}^{\prime}\right)\right]+\Omega \phi_{z^{\prime} z^{\prime}}^{\prime},
\end{aligned}
$$

convenient for applying of a perturbation theory. Let us supply these equations with the boundary conditions:

$w^{\prime}\left(x^{\prime}, z^{\prime}=0, t^{\prime}\right)=w^{\prime}\left(x^{\prime}, z^{\prime}=h, t^{\prime}\right)=0$,

where $h$ is the wave-guide height. It is possible to give various physical interpretations of the boundary conditions imposed. Keeping in mind the atmospheric waves, then the lower boundary condition takes into account impermeability of the Earth's surface. The upper boundary condition qualitatively takes into account the wave reflection effect taking place through diminution of $H(z)$ at the heights $80-90 \mathrm{~km}$ in the real Earth atmosphere. In reality, this wave reflection is not full and is not essential for each wave mode. We somewhat overstate this effect. Selecting the boundary conditions, we not only took into account the conditions of the real atmosphere, but also made an effort to provide an analytical solvability of the nonlinear problem for initial conditions of a rather broad class.

We hope that the essence of many nonlinear effects is determined by the equation structure, and is not dramatically dependent on boundary conditions. If we had used some other boundary conditions, ensuring wave-guide wave propagation, then we would deduce some analogous model equations conterminous in letter with (A3). At last, with our model we might simulate free wave propagation in semiinfinite space as well. In this case, we should lift the upper boundary a little higher, so that this boundary has not enough time to influence the processes taking place near the ground. Such a method is quite admissible for finite times.

To simplify writing, we further omit the primes at the dimensionless variables. Supposing $\sigma=\beta=v=\Omega=0$ in (A1), we obtain the equations of a principal approximation. At this approximation, a general solution to the problem can be constructed with the help of a Fourier method of the separation of variables. As this method is widely known, we do not describe the calculations, but rather write down the outcome in some special form, as a sum of right-hand and left-hand waves:

$u=\sum_{n=1}^{\infty} u^{n}(x, z, t)+\sum_{n=-1}^{-\infty} u^{n}(x, z, t)$, 
$w=\sum_{n=1}^{\infty} w^{n}(x, z, t)+\sum_{n=-1}^{-\infty} w^{n}(x, z, t)$,

$\phi=\sum_{n=1}^{\infty} \phi^{n}(x, z, t)+\sum_{n=-1}^{-\infty} \phi^{n}(x, z, t)$,

$\psi=\sum_{n=1}^{\infty} \psi^{n}(x, z, t)+\sum_{n=-1}^{-\infty} \psi^{n}(x, z, t)$,

$u^{n}(x, z, t)=\Theta^{n}(x, t)$

$$
\cdot\left(S_{n}(z) A_{1, n}+B_{1, n} S_{n}^{\prime}(z)\right) \exp \left(\frac{z}{2}\right),
$$

$w^{n}(x, z, t)=-\frac{\partial}{\partial x} \Theta^{n}(x, t) \cdot c_{n} S_{n}(z) \exp \left(\frac{z}{2}\right)$,

$\phi^{n}(x, z, t)=\Theta^{n}(x, t)$

$$
\cdot\left(S_{n}(z) A_{2, n}+B_{2, n} S_{n}^{\prime}(z)\right) \exp \left(\frac{z}{2}\right),
$$

$\psi^{n}(x, z, t)=\Theta^{n}(x, t)$

$$
\cdot\left(S_{n}(z) A_{3, n}+B_{3, n} S_{n}^{\prime}(z)\right) \exp \left(\frac{z}{2}\right),
$$

$A_{1, n}=\frac{c_{n}}{2}\left(1-\frac{2-c_{n}^{2}}{\gamma-c_{n}^{2}}\right)$,

$A_{2, n}=-\frac{2-c_{n}^{2}}{\gamma-c_{n}^{2}} \cdot \frac{\gamma-1}{2}$,

$B_{1, n}=\frac{\gamma c_{n}}{\gamma-c_{n}^{2}}, \quad B_{2, n}=\frac{c_{n}^{2}}{\gamma-c_{n}^{2}}$,

$A_{3, n}=1-\frac{2-c_{n}^{2}}{\gamma-c_{n}^{2}}, \quad B_{3, n}=\frac{c_{n}^{2}}{\gamma-c_{n}^{2}}$,

$c_{n}=\sqrt{4 \frac{\gamma-1}{\gamma} \frac{1}{1+4 k_{n}^{2}}}, \quad k_{n}>0$,

$c_{-n}=-c_{n}, \quad k_{n}=\frac{n \pi}{h}$,

$S_{n}(z)=\sin k_{n} z, \quad S_{n}^{\prime}=\frac{d S_{n}(z)}{d z}$,

Here the functions $\Theta^{n}$ satisfy the hyperbolic equation

$\Theta_{t}^{n}+c_{n} \Theta_{x}^{n}=0$.

The wave modes with positive numbers are waves propagating to the right, and the wave modes with negative numbers are waves propagating to the left. The vertical structure of each wave mode is fixed, but the solution as a whole takes into account a vertical propagation of waves.

When $\sigma \neq 0, \beta \neq 0, v \neq 0, \Omega \neq 0$, equations (A1) are nonlinear ones. Because of $\sigma \ll 1, \beta \ll 1, v \ll 1, \Omega \ll 1$, the right-hand sides of the equations (A1) are small ones. It is possible to spread out the description of wave processes in terms of wave modes to nonlinear case. We shall calculate it with the help of a Galerkin method, combining this method with a perturbation theory. A Galerkin method uses the expansion of a desired solution into a series of a complete set of functions. The choice of the complete set of functions used is almost unrestricted. It is advantageous to keep a wave mode concept in the nonlinear theory. Therefore, we shall use those eigenfunctions of $z$ which have arisen in the problem with $\sigma=\beta=v=\Omega=0$. That is, we will use the vector-functions

$$
\left(\begin{array}{c}
\left(S_{n}(z) A_{1, n}+B_{1, n} S_{n}^{\prime}(z)\right) \exp \left(\frac{z}{2}\right) c_{n} \\
c_{n} S_{n}(z) \exp \left(\frac{z}{2}\right) \\
\left(S_{n}(z) A_{2, n}+B_{2, n} S_{n}^{\prime}(z)\right) \exp \left(\frac{z}{2}\right) \\
\left(S_{n}(z) A_{3, n}+B_{3, n} S_{n}^{\prime}(z)\right) \exp \left(\frac{z}{2}\right)
\end{array}\right)
$$

of $z$ as a basis for expansion of the desired solution

$$
\left(\begin{array}{l}
\psi(x, z, t) \\
u(x, z, t) \\
w(x, z, t) \\
\varphi(x, z, t)
\end{array}\right)
$$

into a Fourier series.

Let $\Theta^{n}(x, t)$ denote the series coefficients. In this way, we search for a solution of the nonlinear problem in form (A2), similar to a linear theory. Now, however, the functions $\Theta^{n}(x, t)$ have to satisfy some nonlinear equations. Within the framework of a Galerkin method, the derivation of equations for $\Theta^{l}(x, t)$ is based on the orthogonality relations for basis functions. The wave modes $\left(\begin{array}{c}u^{n} \\ w^{n} \\ \phi^{n} \\ \psi^{n}\end{array}\right)$ and $\left(\begin{array}{c}u^{m} \\ w^{m} \\ \phi^{m} \\ \psi^{m}\end{array}\right)$ are orthogonal to each other for $m \neq n$ in the sense that

$$
\begin{aligned}
& \left\langle\begin{array}{cc}
u^{n} & u^{m} \\
w^{n} & w^{m} \\
\phi^{n} & , \\
\phi^{m} \\
\psi^{n} & \psi^{m}
\end{array}\right\rangle=\int_{-\infty}^{\infty} \int_{0}^{h}\left(u^{n} u^{m}+\beta^{2} w^{n} w^{m}+\right. \\
& \left.\quad+\phi^{n} \phi^{m} \frac{1}{\gamma-1}+\psi^{n} \psi^{m}\right) \exp -\frac{z}{2} d z d x=0
\end{aligned}
$$

at $n \neq m$. Here the design $\langle\cdot, \cdot\rangle$ on the left denotes the scalar product introduced and the definition of the scalar product is written out on the right.

At first, we substitute initial objects ( A2) into (A1). Then we multiply the first equation by $\psi^{l}$, the second equation by $u^{l}$, the third equation by $w^{l}$, and the fourth by $\phi^{l}(\gamma-$ $1)^{-1}$. The results are multiplied by $\exp (-z / 2)$. Then we add together the outcomes and integrate over $x$ from $-\infty$ up to $\infty$ and over $z$ from zero up to $h$. The operations made are equivalent to scalar multiplication of equations (A1) by $\left(\begin{array}{c}u^{l} \\ w^{l} \\ \phi^{l} \\ \psi^{l}\end{array}\right)$. Let us calculate the integrals over $z$. With the help of integrating by parts with respect to $x$ we come to

$$
\begin{array}{r}
\int_{-\infty}^{\infty} \Theta^{l}\left(\Theta_{t}^{l}+c_{l} \Theta_{x}^{l}+\frac{\sigma}{2} \sum_{m, l} A_{m, n}^{l} \Theta^{n} \Theta_{x}^{m}\right. \\
\left.+\frac{\sigma}{2} \sum_{m, n} B_{m, n}^{l} \Theta^{m} \Theta_{t}^{n}+\ldots\right) d x=0
\end{array}
$$


Here the symbols $c_{l}, A_{m, n}^{l}, B_{m, n}^{l}$ denote some constants which have arisen after integration over $z$. Because of arbitrary dependence of $\Theta^{l}$ on $x$, the term within the square brackets is equal to zero.

We have obtained some equations. These equations are practically equivalent to the original hydrodynamic equations. (If we did not take into consideration dissipation effects, the equations would be equivalent.) It is useful to modify slightly these equations. We see that the relation $\Theta_{t}^{l}+c_{l} \Theta_{x}^{l} \approx 0$ is valid. We shall use this relation in the form $\Theta_{t}^{l} \approx-c_{l} \Theta_{x}^{l}$ to exclude all small terms with timederivatives. For example, $\beta^{2} \Theta_{t t x} \approx \beta^{2} c_{l}^{2} \Theta_{x x x}$. Therefore, we obtain the set of equations:

$$
\begin{aligned}
\Theta_{t}^{l}+ & c_{l} \Theta_{x}^{l}+\frac{\sigma}{2} \sum_{m, l} F_{n, m}^{l} \Theta^{n} \Theta_{x}^{m} \\
& +\frac{1}{2} \beta^{2}\left(\frac{\gamma-c_{l}^{2}}{\gamma-1} c_{l}^{3} \Theta_{x x x}^{l}+\frac{\gamma+c_{l}^{2}}{\gamma-1} c_{l}^{3} \Theta_{x x x}^{-l}\right) \\
& +\frac{1}{2} v_{0} \sum_{n} K_{n}^{l} \Theta^{n}=0 .
\end{aligned}
$$

The small parameter $v_{0}=\sup _{0 \leq z \leq h}(\Omega(z), v(z))$ is entered for convenience, in order that $K_{n}^{\bar{l}}=O(1)$. The factors $F_{k, m}^{l}, K_{n}^{l}$ are cumbersome ones, and consequently they are not written here. They are readily calculated with the help of any program of analytical evaluations.

The $F_{k, m}^{l}, K_{n}^{l}$ are coefficients of Fourier series as well; and, at variation of indexes, they behave as regular Fourier series coefficients. If all indexes are fixed, except one, and if this one selected index tends towards infinity, then the coefficients $F_{k, m}^{l}, K_{m}^{l}$ will not decrease more slowly than inversely to this index. The functions $\Theta^{l}$ are nothing else but coefficients of a generalised Fourier series. Therefore they have to decrease at $l \rightarrow \infty$ as well. Hence, one can break off the set of equations, taking into consideration perhaps a lot, but a finite number of wave modes. Being prudent enough, we can break off the line-up of equations, even if only a few wave modes were originally excited. At such a breaking off, we neglect the effects of the mutual generation of wave modes. In particular, if we neglect dissipation effects and if we take into account only one wave mode, we shall obtain a $\mathrm{KdV}$ model of internal waves (Leonov, 1976; Ostrovskiy, 1979, 1986; Segur and Hammack, 1982).

With the error $O\left(\sigma^{2}+v_{0}^{2}+\beta^{4}\right)$, the equations deduced are equivalent to the primitive hydrodynamic equations. However, some boundary effects stipulated by viscosity and thermal conductivity are not taken into consideration because we used the basis of a nonviscous problem. In addition, we have excluded acoustic waves from consideration. They were eliminated when we had used the relation $\Theta_{t}^{l} \approx-c_{l} \Theta_{x}^{l}$ for the simplification of the terms about $\beta^{2}$.

An approximate solution to (A3) can be constructed with the help of a nonsingular perturbation theory. The approximate solution is constructed as follows. At first, a usual perturbation theory series in parameters $\sigma, \beta^{2}, v_{0}$ is written down. Evidently, in the first order of the perturbation theory we have the problem with $\sigma=\beta=\nu_{0}=0$. Its general solution is $\Theta^{l}(x, t)=\Theta_{0}^{l}\left(x-c_{l} t\right)$. In the following order of the perturbation theory, the corrections proportional $\sigma, \beta^{2}$, $\nu_{0}$ are taken into account. Some of these corrections are secular ones at $t \rightarrow \infty$; they grows as $t$ grows. Hence the usual perturbation theory is usable only for time spans of $O(1)$. To get rid of the secular terms in the perturbation theory, the equation terms generating the secular terms of the perturbation theory are taken into consideration in the starting order of a new perturbation theory. Then the starting equations become more complicated ones, but the new perturbation theory gives an approximate solution applicable for long $t$. This approximate solution is

$$
\begin{aligned}
& \Theta^{l}(x, t) \asymp \Theta_{0}^{l}(x, t)-\int_{0}^{t}\left[\frac{\sigma}{2} \sum_{\substack{m, n \\
m \neq n \neq l}}\right. \\
& F_{n, m}^{l} \Theta_{0}^{n}\left(x-c_{l}\left(t-t^{\prime}\right), t^{\prime}\right) \Theta_{0 x}^{m}\left(x-c_{l}\left(t-t^{\prime}\right), t^{\prime}\right) \\
& +\frac{\beta^{2}}{2} \frac{\gamma+c_{l}^{2}}{\gamma-1} c_{l}^{3} \Theta_{x x x}^{-l}\left(x-c_{l}\left(t-t^{\prime}\right), t^{\prime}\right) \\
& \left.+\frac{\nu_{0}}{2} \sum_{m \neq l} K_{m}^{l} \Theta_{0}^{m}\left(x-c_{l}\left(t-t^{\prime}\right), t^{\prime}\right)\right] d t^{\prime}
\end{aligned}
$$

Here the functions $\Theta_{0}^{l}(x, t)$ are solutions of independent Korteweg-de Vries equations with damping

$$
\begin{aligned}
\Theta_{0 t}^{l}+ & c_{l} \Theta_{0 x}^{l}+\frac{\sigma}{2} F_{n, m}^{l} \Theta_{0}^{l} \Theta_{0 x}^{l} \\
& +\frac{\beta^{2}}{2}\left(\frac{\gamma-c_{l}^{2}}{\gamma-1}\right) c_{l}^{3} \Theta_{0 x x x}^{l}+\frac{1}{2} v_{0} K_{l}^{l} \Theta_{0}^{l}=0
\end{aligned}
$$

The initial conditions are posed so: $\Theta_{0}^{l}(x, 0)=\Theta^{l}(x, 0)$.

The first term of the integrand in (A4) takes into account nonlinear interaction of various modes. The addend of this integrand takes into account the "dispersion-stipulated" interaction with the wave propagating in the opposite direction. In fact, this addend may be excluded from (A4), having made some small suitable corrections in the initial functions $\Theta_{0}^{l}(x, 0)$. The last term of the integrand takes into account the interaction of various modes through dissipation. This interaction takes place because the basis functions utilized are not eigen-functions to the dissipative problem.

The quality of approximation (A4), (A5) was checked by means of comparison of these formulas with the numerical solutions of (A3) (Kshevetskii and Leble, 1985, 1988). Satisfactory concurrence of the analytical formula to the numerical outcomes was shown.

We see that the interactions of various modes are insignificant in the principal order; these effects are of the following order of smallness. For this reason, a simple $\mathrm{KdV}$ model is effective. The investigation of various mode interactions was not planned in the present paper; we describe these effects only briefly. The interactions of various wave modes result in phase shifts of collided solitons and in feeble energy interchange between wave modes (Kshevetskii and Leble, 1985 , 1988). Phase shifts of collided solitons were computed in 
Kshevetskii and Leble, $(1985,1988)$. The effects of the interaction of wave modes $n, m$ are proportionate to $\sigma /\left(c_{m}-c_{n}\right)$. For higher modes the residuals $\left|c_{m}-c_{n}\right|$ become small ones. Therefore, the effects of the interaction of various modes can be considerable for higher modes, and then the approximate solution constructed loses a force. Equations (A3), however, are valid in any case.

\section{Appendix B Development of the KdV model of nonlin- ear internal waves}

The roots of the analytical model under consideration lies in the pioneer paper by Long. In 1965, Long investigated the propagation of stationary, weakly nonlinear long internal waves in a wave duct filled with an incompressible fluid stratified by gravity. In a linear long wavelength approximation the wave considered was described by the formula $\Theta^{n}\left(x-c_{n} t\right) \cdot S^{n}(z)$. Here $c_{n}$ is the wave speed. In this approximation, there are no restrictions on the dependence of $\Theta^{n}$ on $\left(x-c_{n} t\right)$. The function $S^{n}(z)$ describes the vertical wave structure. The function $S^{n}(z)$ and parameter $c_{n}$ are an eigenfunction and eigenvalue of some Sturm-Liouville boundary value problem. We shall not write out this problem here, as its exact shape is not important. The function $\Theta^{n}\left(x-c_{n} t\right)$ describes the horizontal wave structure. The Sturm-Liouville problem has an innumerable set of solutions, consequently all functions are supplied with indexes. The index shows the wave mode number. Long has shown that, if we take into consideration weak effects of nonlinearity and dispersion, the function $\Theta^{n}\left(x-c_{n} t\right)$ of argument $\left(x-c_{n} t\right)$ now does not stay be any. Its form is governed by the stationary Korteweg-de Vries equation:

$\left(\tilde{c}_{n}-c_{n}\right) \Theta_{\xi}^{n}+\sigma a^{n} \Theta^{n} \Theta_{\xi}^{n}+\beta^{2} b^{n} \Theta_{\xi \xi}^{n}=0$.

Here parameter $\tilde{c}_{n}$ denotes the nonlinear wave propagation velocity, $\xi=x-\tilde{c}_{n} t, \tilde{c}_{n}>c_{n}, \sigma$ and $\beta$ are small amplitude and dispersion parameters. The constants $a^{n}, b^{n}$ depend on the considered wave mode. They also depend on the waveguide depth and density stratification scale. The influence of nonlinear effects on the vertical wave structure was not considered by Long.

If we use a soliton solution of equation (B1), we shall obtain a so-called solitary internal gravity wave. Long has made the note that the solitary wave is absent at $\beta=0$. That is, equation (B1) has no physically interpreted solutions for $\beta=0$. Thus, Long for the first time, has made the observation that a quasistatic approach changes radically some solutions to the nonlinear problem, even if long waves are considered.

Later, Benjamin has generalised Long's outcomes to the case of stationary wave propagation in a stratified incompressible fluid with a free surface (Benjamin, 1966). Many papers of interest were published since then. In 1975, Pelinovskiy and Romanova have shown that a stationary Korteweg-de Vries equation describes the propagation of nonlinear internal gravity waves in a stratified gas. Later, Panchev and Evtimov have given other derivation of the stationary $\mathrm{KdV}$ equation for internal gravity waves propagated in the equatorial atmosphere (1978).

In 1976, Leonov has deduced a non-stationary, two-dimensional Korteweg-de Vries equation describing the propagation of one excited wave mode. The horizontal structure of that wave is time-dependent; at initial instant it may be given by any function.

In this appendix, we pursued the purpose to remind the reader of the $\mathrm{KdV}$ model of internal waves. Because of this direction, unfortunately many advanced papers were not mentioned. For example, Rid and Su (1984) investigated the interaction of internal wave solitons. Equations (2) are not only possible generalisations of classical outcomes by Long and Benjamin. Ostrovskiy has deduced some other generalising set of equations. The equations by Ostrovskiy are similar to the equations of a small-depth water (Ostrovskiy, 1986). Gear and Grimshaw have created the KdV model improvement at the expense of taking into account nonlinear terms of the following order of smallness (1983). Meisen, Kamp and Sluijter have deduced a Benjamin-Davis-Ono equation for internal waves (1990). The achievements in the study of ocean soliton waves have been expounded in the in depthreview by Ostrovskiy and Stepanyants (1989). Huthnance has made a review on research of internal tides (1989). Lamb numerically investigated the solitary internal wave generated at intersection by a tidal force stream of a finite amplitude bank edge (Lamb, 1994).

The papers by Koop and Butler (1982), Segur and Hammack (1982) and Buckreev and Gavrilov (1983) are devoted to experimental verification of soliton models of internal waves (KdV, Benjamin-Ono, nonlocal KP and other models). On unanimous inference of the authors of the experiments, the $\mathrm{KdV}$ model is the most exact one. (Only single-mode models were considered.) However, this conclusion has been made under some concrete conditions. It is hardly probable that this conclusion has no exceptions.

Notwithstanding obvious favour of analytical models, they all have some common shortfalls. All the analytical models are asymptotic ones. Roughly speaking, it means that they "work" only in the case where the small parameters of the theories are sufficiently small. The concept of a "sufficient smallness" is vague to some extent, but it is very difficult to deduce an exact estimate of the model error. Now only an experiment can answer the question of applicability of the analytical model to a particular event. A number of simplifying suppositions is another shortfall of analytical models. Some simplifying suppositions are not desirable from the physical point of view, but it fails to advance in analytical modelling without them.

Acknowledgements. The author thanks S. B. Leble and V. V. Shuvalov for arguing of the outcomes. The investigations were supported by the grant 97-0-13.3-72 of Ministry of Education of Russia. 


\section{References}

Benjamin, T., Internal wave of finite amplitude and permanent form, J. Fluid. Mech., 25, 241-270, 1966.

Buckreev, V. I. and Gavrilov, N. V., Experimental research of solitary internal waves in a two-layer fluid, Prikladnaya mechanics i technical physics, 5, 51, 1983.

Cotton, W. R. and Tripoli, G. I., Cumulus convection in shear flow three-dimensional numerical experiments, J. Atmos. Sci., 35, 8, 1503-1521, 1978.

Dikiy, L.A., The theory of oscillations of the terrestrial atmosphere, Hydrometeorological Publishing House, Leningrad, 1969.

Fletcher, C., Computational Galerkin Methods, Springer-Verlag, 1984.

Gear, J. A. and Grimshaw, R., A second order theory for solitary waves in shallow fluids, Phys. Fluids, 26, 1, 14-29, 1983.

Gossard, E. and Hook, U., Waves in the atmosphere, Elsevier Scientific Publishing Company, 1975.

Gürses, M. and Karasu, A., Degenerate Svinolupov KdV systems, Physics Letters A, 214, 21-26, 1996.

Gürses, M. and Karasu, A., Integrable coupled KdV systems, J. Math. Phys., 39, 4, 2103-2111, 1998.

Hurthnance, J. M., Internal tides and waves near the continental shelf edge, Geophys. Fluid Dyn., 48, 81-106, 1989.

Yudin, V. and Gavrilov, N., The algorithm of simulation of gravity wave propagation raised by non- stationary local sources in a stratified atmosphere, Published by the editors of the journal "Izvestiya Academy Nauk SSSR. Fizika atm. okeana", VINITI N 2865-85, 1985.

Klemp, J. B. and Wilhelmson, R. B., Simulation of threedimensional convective storm dynamics, J. Atmos. Sci., 35, 6, 1079-1096, 1978.

Koop, C. G. and Butler, G., An investigation of internal solitary waves in a two-fluid system, J. Fluid Mech, 112, 225, 1982.

Kshevetskiy, S., The numerical methods of solving of Euler equations for heavy liquid. The solitons simulation, International Symposium on Nonlinear Theory and Its Applications (NOLTA 95), Las-Vegas, USA, 1995.

Kshevetskii, S., Comparison between an analytic model of nonlinear internal waves and the results of numerical experiments, Izvesiya, Atmospheric and Oceanic Physics, 34, 3, 282, 1998.

Kshevetskii, S. and Leble, S. B., Nonlinear dispersion of large-scale internal waves, Izvesiya, Atmospheric and Oceanic Physics, 21, 5, 1169-1174, 1985.

Kshevetskii, S. and Leble, S. B., Nonlinear dispersion of long internal waves, Fluid dynamics, 23, 448-452, 1988.

Kshevetskii, S., Zacharov, L., and Sergevs, S., Linear nonhydrostatic numerical models of internal gravity wave propagation in the atmosphere, Published by the editors of the journal "ZVMiMF", VINITI N3834-B90, 1990.

Lamb J. L., Jr., Elements of soliton theory, John Wiley and Sons,
Inc., 1980.

Lamb, K. G., Finite amplitude bank edge, J. Geophys. Res., 99, 843-864, 1994

Leonov, A. I. On the two-dimensional Korteweg-de Vries equation in the theory of nonlinear surface and internal waves, Dokl. AN SSSR, 229, 4, 820-824, 1976.

Lions, J. L., Quelques methodes de resolution des problemes aux limites non linearies, Paris, 1969.

Long, R. R., On the Boussinesque approximation and its role in the theory of internal waves, Tellus, 17, 1965.

McEwan, A. D., The kinematics of stratified mixing through internal wavebreaking, J. Fluid Mech., 128, 47-57, 1983.

Meisen, R. H., Kamp, L. P. J., and Sluijter F. W., Solitary waves in compressible deep fluids, Phys. Fluids, A2, 8, 1434-1411, 1990.

von Neumann, J. and Richtmyer, R. D., A method for the numerical calculation of hydrodynamic shocks, J. Appl. Physics, 21 , 232 237,1950

Ostrovskiy, L. A., Nonlinear internal waves in the ocean, Nonlinear waves, Moscow, 292, 1979.

Ostrovskiy, L. A., Internal wave in the ocean: the theory and observation, 1987 Materials Vsesouznoy conference, Solnechnogorsk, March 1986.

Ostrovskiy, L. A. and Stepanyants, Yu. A., Do internal solitons exist in the ocean? Reviews Geophys., 27, 293-310, 1989.

Panchev, S. and Evtimov, St., On stationary nonlinear waves in the equatorial atmosphere, Bulgar. Academy of Sciences. Bulg. Geophysical Journal, 4, 2, 1978.

Peckelis, V. M., The schemes of variable directions and their application to the problems of local weather forecast. Problems on hydrometeorological short-term weather forecast and mezometeorology, The Laboratory of Gidrometeocentre of the USSR, Gidrometeoizdat, Leningrad, 298, 23-73, 1988.

Pelinovskiy, E. N. and Romanova, N. N., Nonlinear stationary waves in the atmosphere, Izvesiya, Atmospheric and Oceanic Physics, 13, 11, 1169-1174, 1975.

Penencko, B. and Aloyan, A., Models and methods for problems of environment protection, Novosibirsk, Nauka, 1985.

Richardson, L. F. Weather prediction by numerical process, Cambridge University Press, Cambridge, 1922.

Richtmyer, R., Principles of modern mathematical physics, v. 1, Springer-Verlag, 1978.

Richtmyer, R. and Morton, K., Difference methods for solving of initial problems, Interscience publishers, 1967.

Mirie Rida M. and Su, C. H., Internal solitary waves and their headon collision, Part 1, J. Fluid Mech., 147, 213-231, 1984.

Samarskiy, A. A. and Popov, Yu. P. Difference methods to solve gas dynamics problems, Moscow, Science, 1980.

Segur, H. and Hammack, J. L., Solitons models of long internal waves, J. Fluid Mech., 118, 285, 1982.

Tapp, M. C. and White, R. W., A nonhydrostatic mesoscale model. Quart. J. Roy. Met. Soc., 102, 432, 277-296, 1976. 